Supporting Information

\title{
Synthesis of Naphthaleman Family Utilizing Regiocontrolled Benzannulation: Unique Molecules Composed of Multi-substituted
}

\section{Naphthalenes}

Sekiha Ishikawa, Yoshikazu Masuyama, Takeshi Adachi, Takeshi Shimonishi, Shotaro Morimoto, Yoo Tanabe*

Department of Chemistry, School of Science and Technology, Kwansei Gakuin University, 2-1 Gakuen, Sanda, Hyogo, 669-1337, Japan

\section{tanabe@kwansei.ac.jp}

\section{Table of Contents}

The title page (Page S1)

${ }^{1} \mathrm{H}$ NMR and ${ }^{13} \mathrm{C}$ NMR spectra for all new compounds 5, 6, 7a-7d, 9, 10, 1a-1d, 14, 15, 16 (Pages S2-S16)

Charts of HPLC analyses for compound 1d (racemic) (Page S17)

Table 1: Optimization of PEPPSI ${ }^{\mathrm{TM}}-\mathrm{IPr}-\mathrm{K}_{2} \mathrm{CO}_{3}$ catalysis using 6 (Page S18)

Data using Spartan software (Wavefunction, Inc. ver. '18 1.1.0) of 1a and 1d (Pages S19-S27)

Corresponding Author: Professor Yoo Tanabe

Department of Chemistry, School of Science and Technology, Kwansei Gakuin University, 2-1 Gakuen, Sanda, Hyogo, 669-1337, Japan 


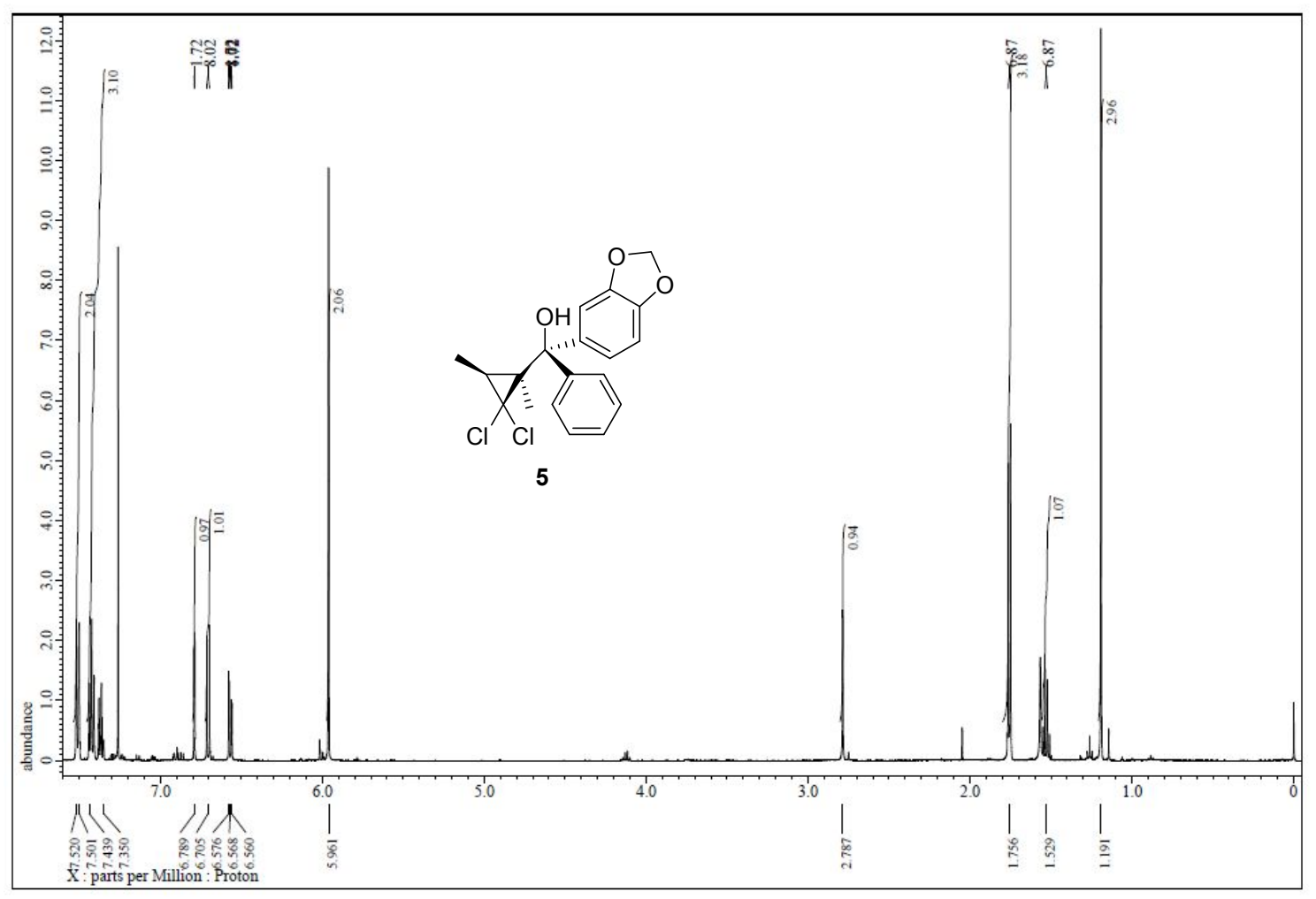

Figure S1: ${ }^{1} \mathrm{H}$ NMR (500 MHz, $\left.\mathrm{CDCl}_{3}\right)$ Spectrum of the Compound 5

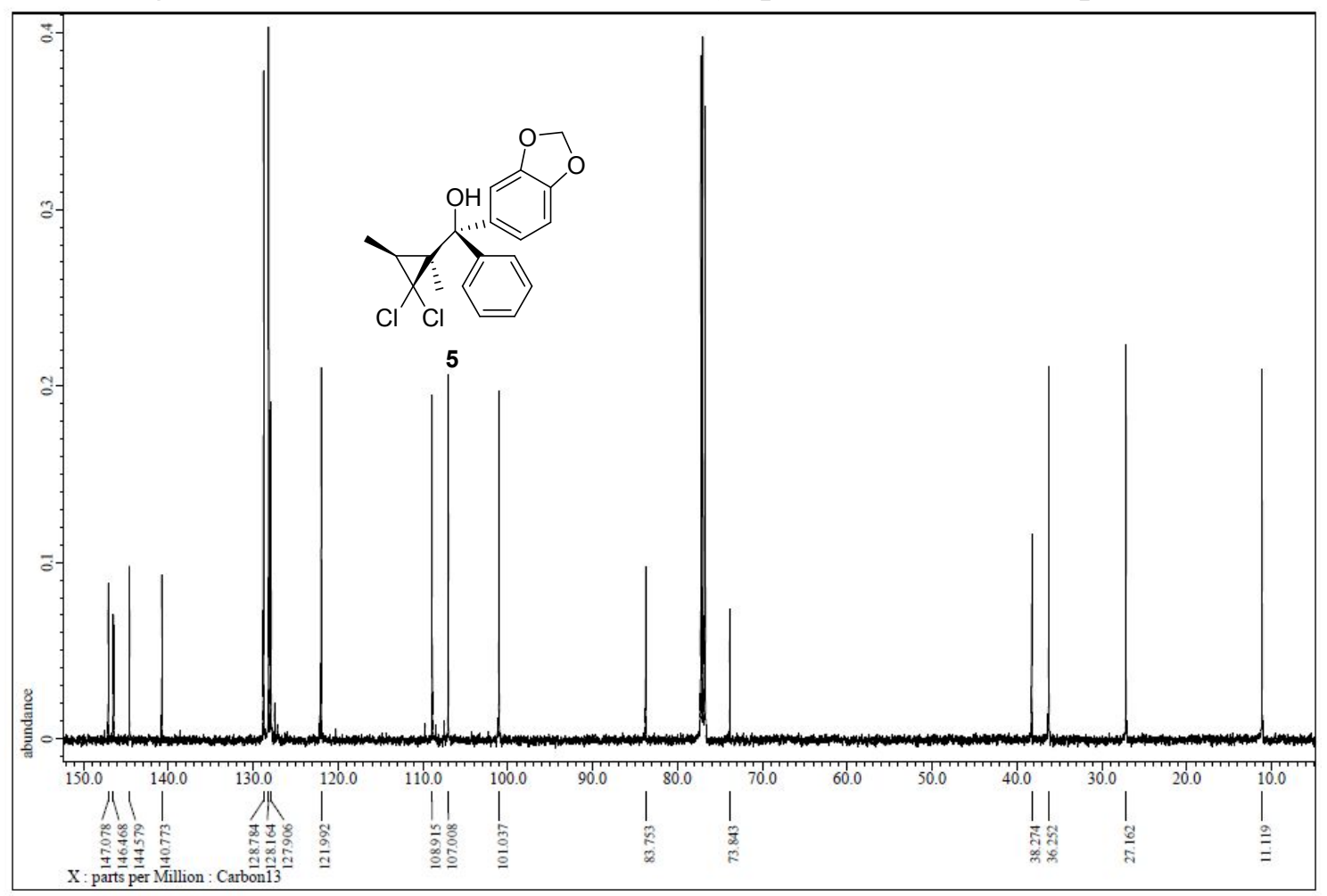

Figure S2: ${ }^{13} \mathrm{C}$ NMR (125 $\left.\mathrm{MHz} \mathrm{CDCl}_{3}\right)$ Spectrum of the Compound 5 


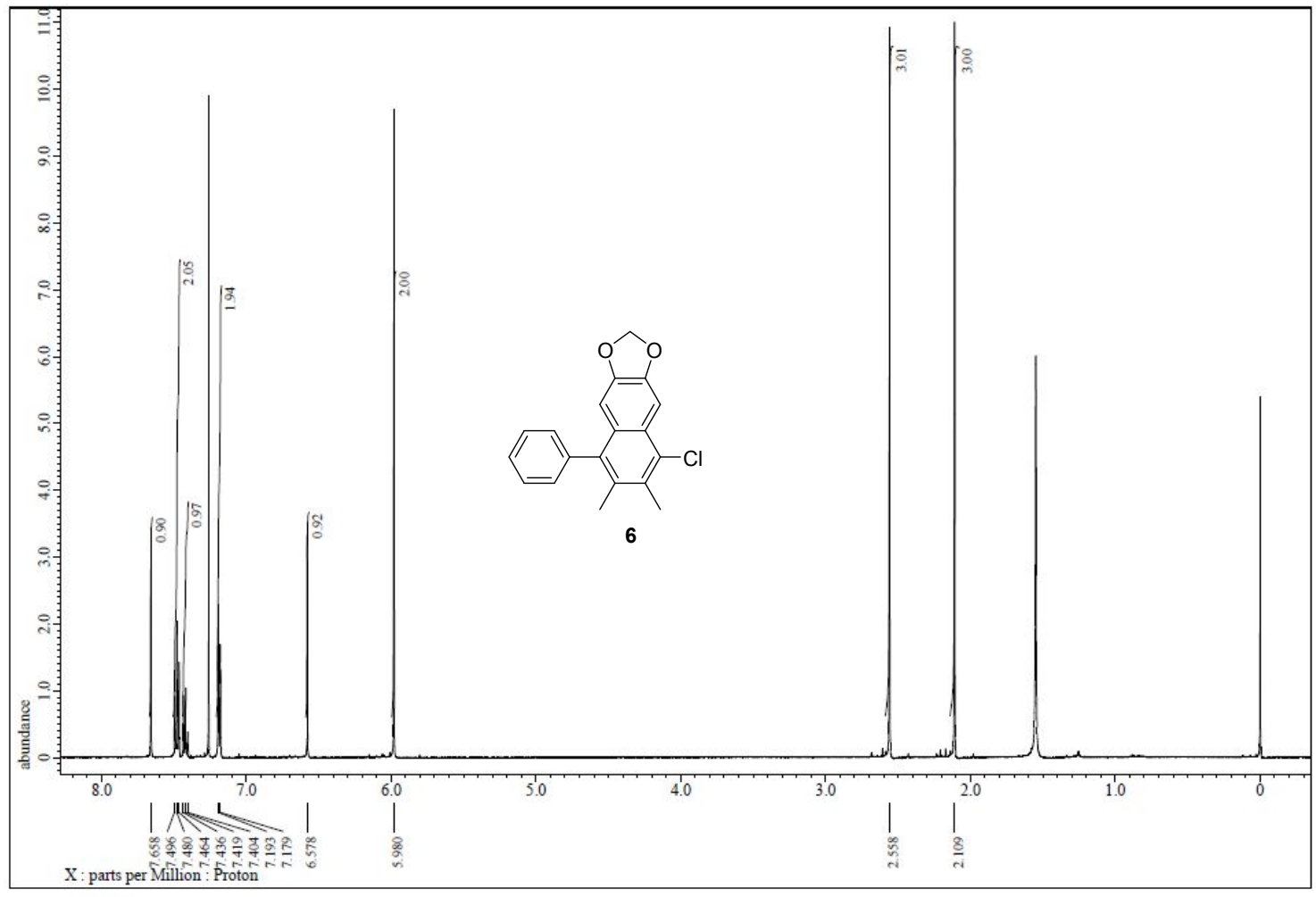

Figure S3: ${ }^{1} \mathrm{H}$ NMR $\left(500 \mathrm{MHz}, \mathrm{CDCl}_{3}\right)$ Spectrum of the Compound 6

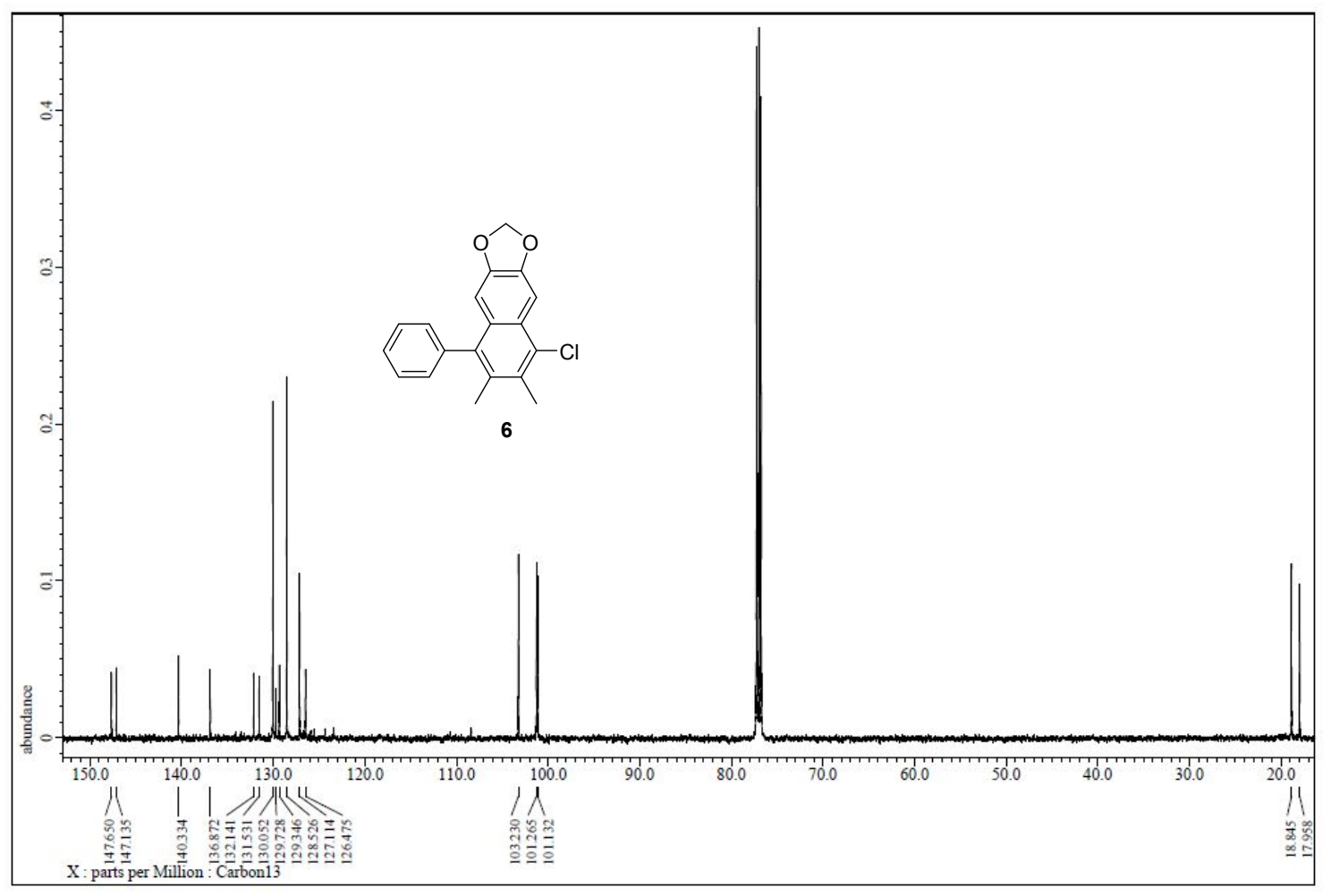

Figure S4: ${ }^{13} \mathrm{C}$ NMR (125 $\left.\mathrm{MHz} \mathrm{CDCl}_{3}\right)$ Spectrum of the Compound 6 


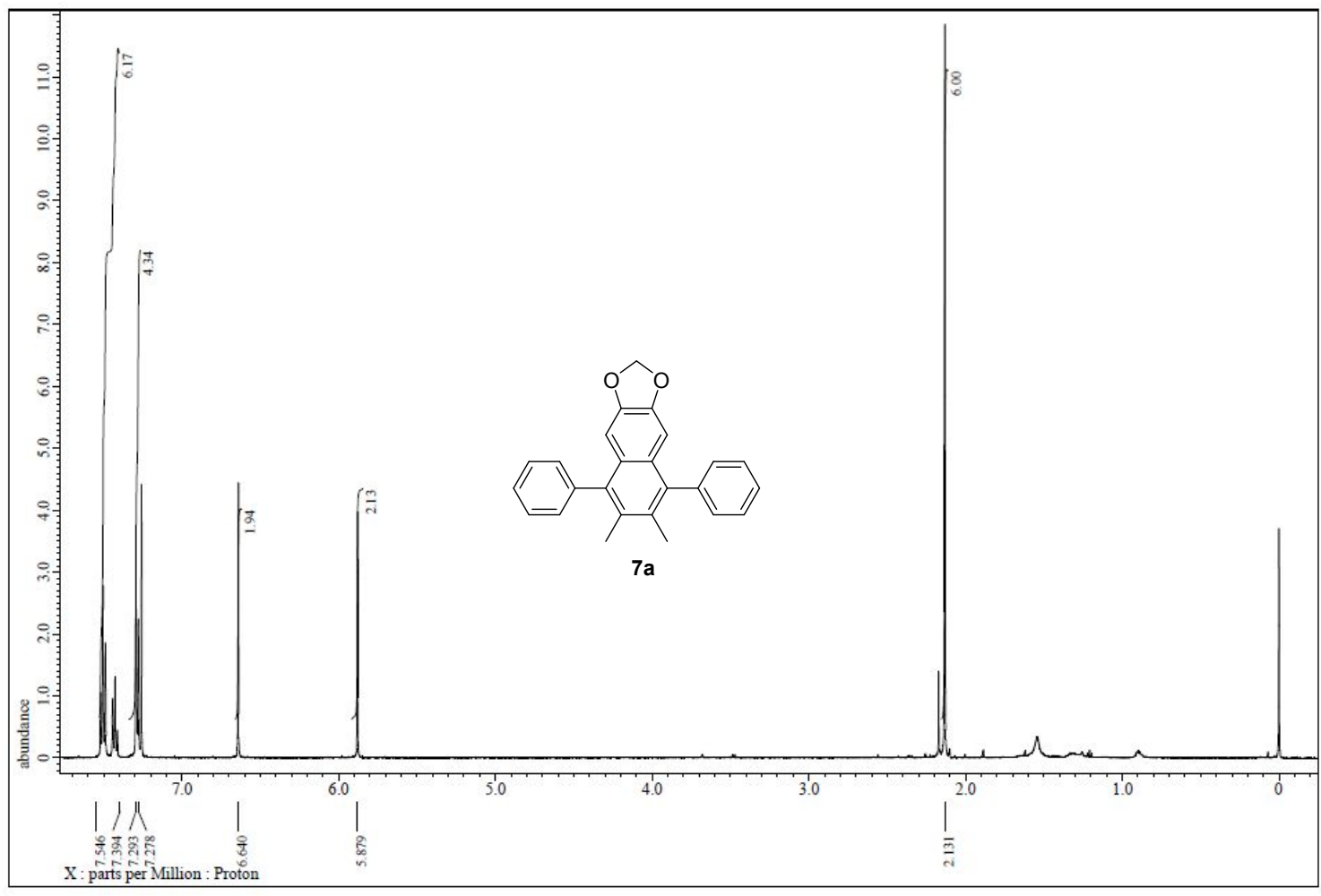

Figure S5: ${ }^{1} \mathrm{H}$ NMR (500 MHz, $\left.\mathrm{CDCl}_{3}\right)$ Spectrum of the Compound 7a

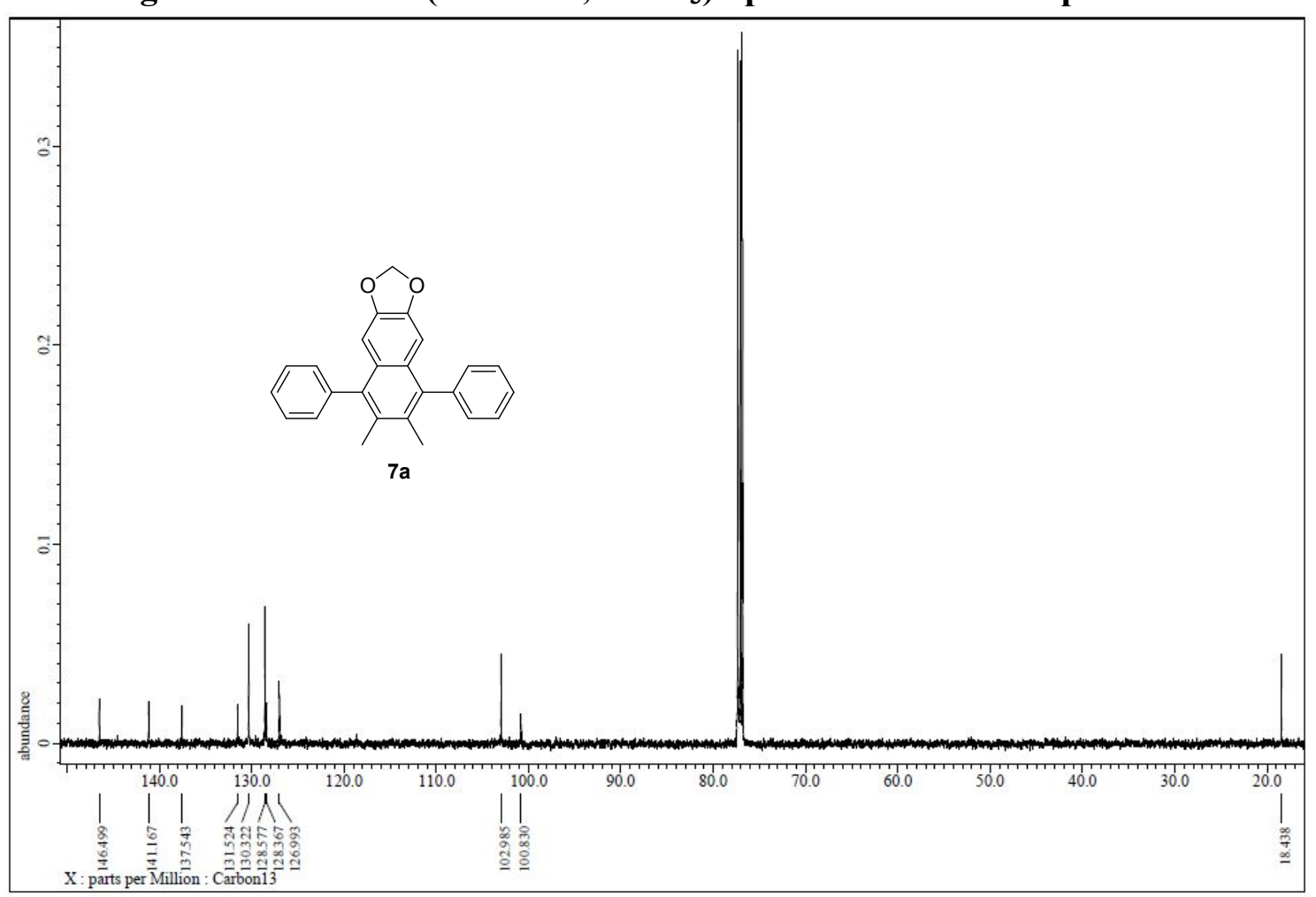

Figure S6: ${ }^{13} \mathrm{C}$ NMR (125 MHz, $\left.\mathrm{CDCl}_{3}\right)$ Spectrum of the Compound 7a 


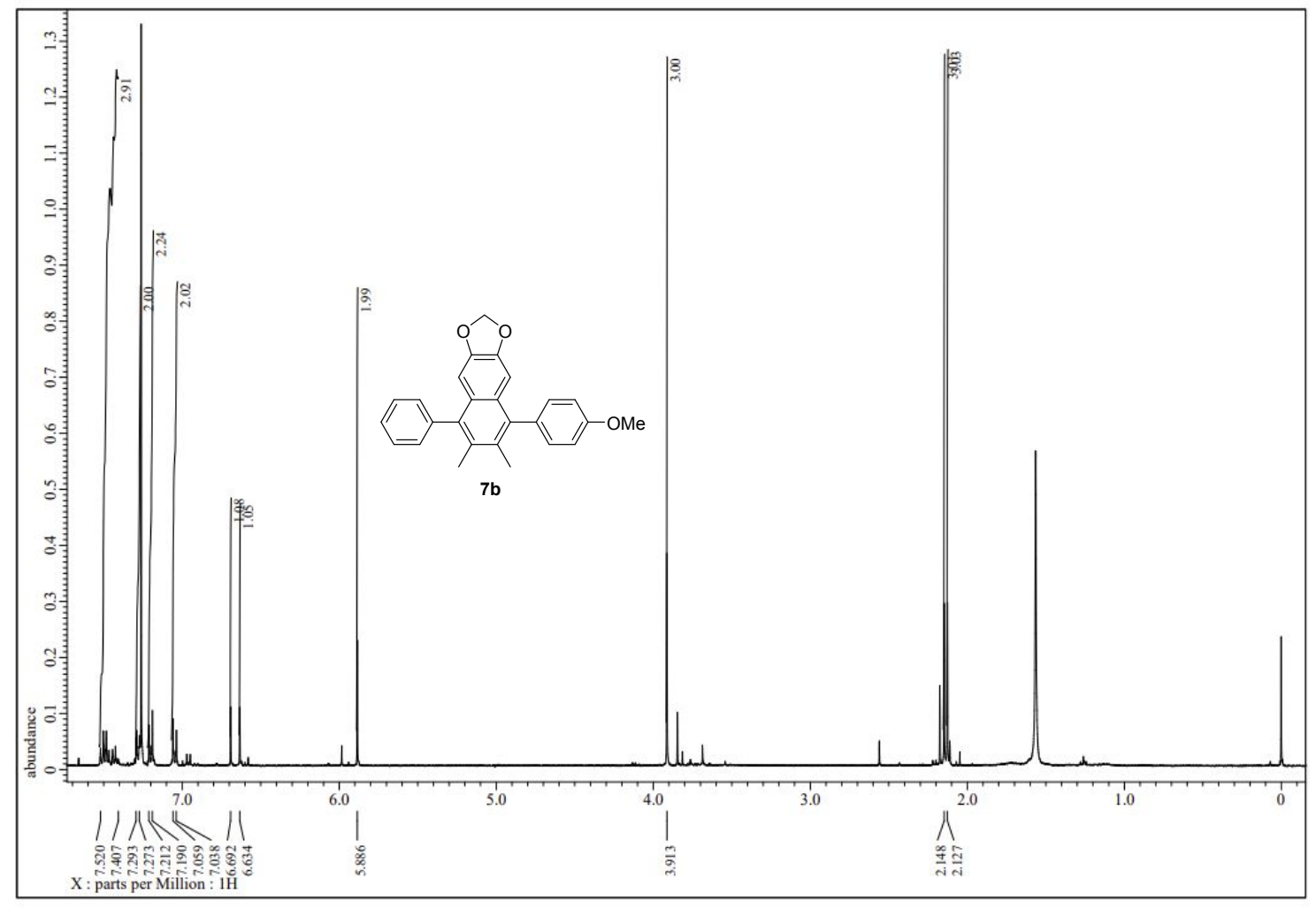

Figure S7: ${ }^{1} \mathrm{H}$ NMR (400 $\left.\mathrm{MHz}, \mathrm{CDCl}_{3}\right)$ Spectrum of the Compound 7b

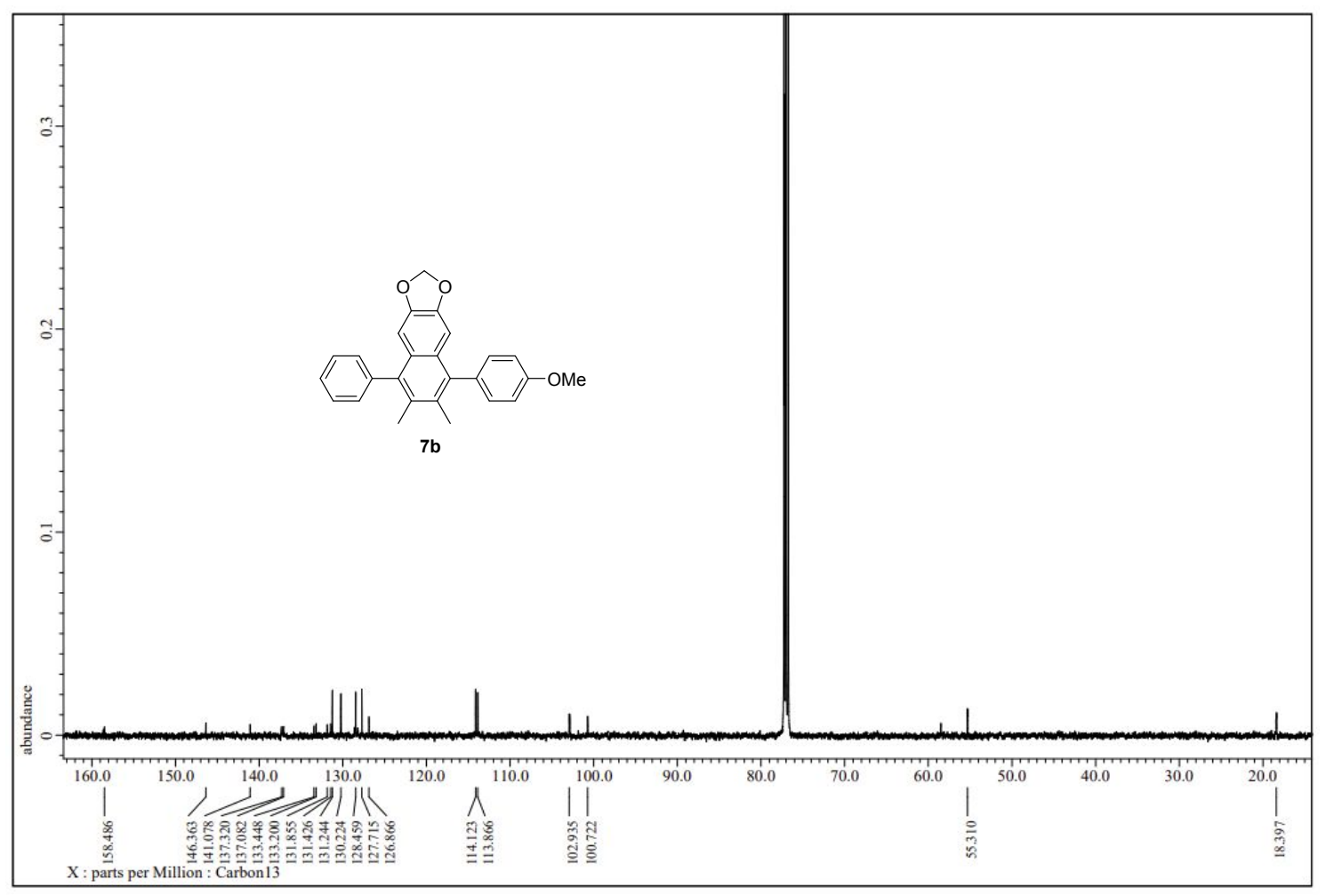

Figure S8: $\left.{ }^{13} \mathrm{C} \mathrm{NMR} \mathrm{(100} \mathrm{MHz,} \mathrm{CDCl}_{3}\right)$ Spectrum of the Compound $7 \mathrm{~b}$ 


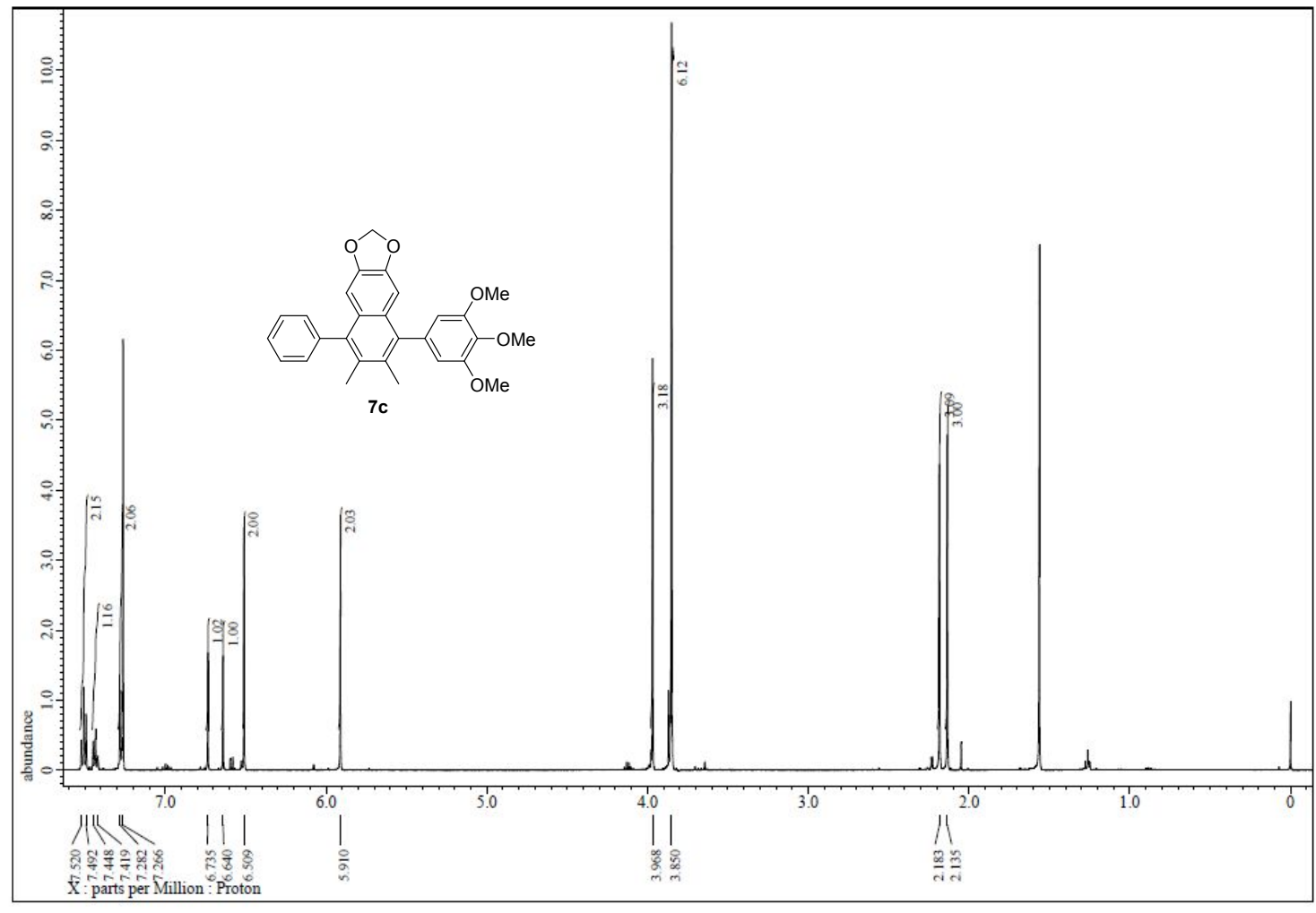

Figure S9: ${ }^{1} \mathrm{H}$ NMR (500 $\left.\mathrm{MHz}, \mathrm{CDCl}_{3}\right)$ Spectrum of the Compound 7c

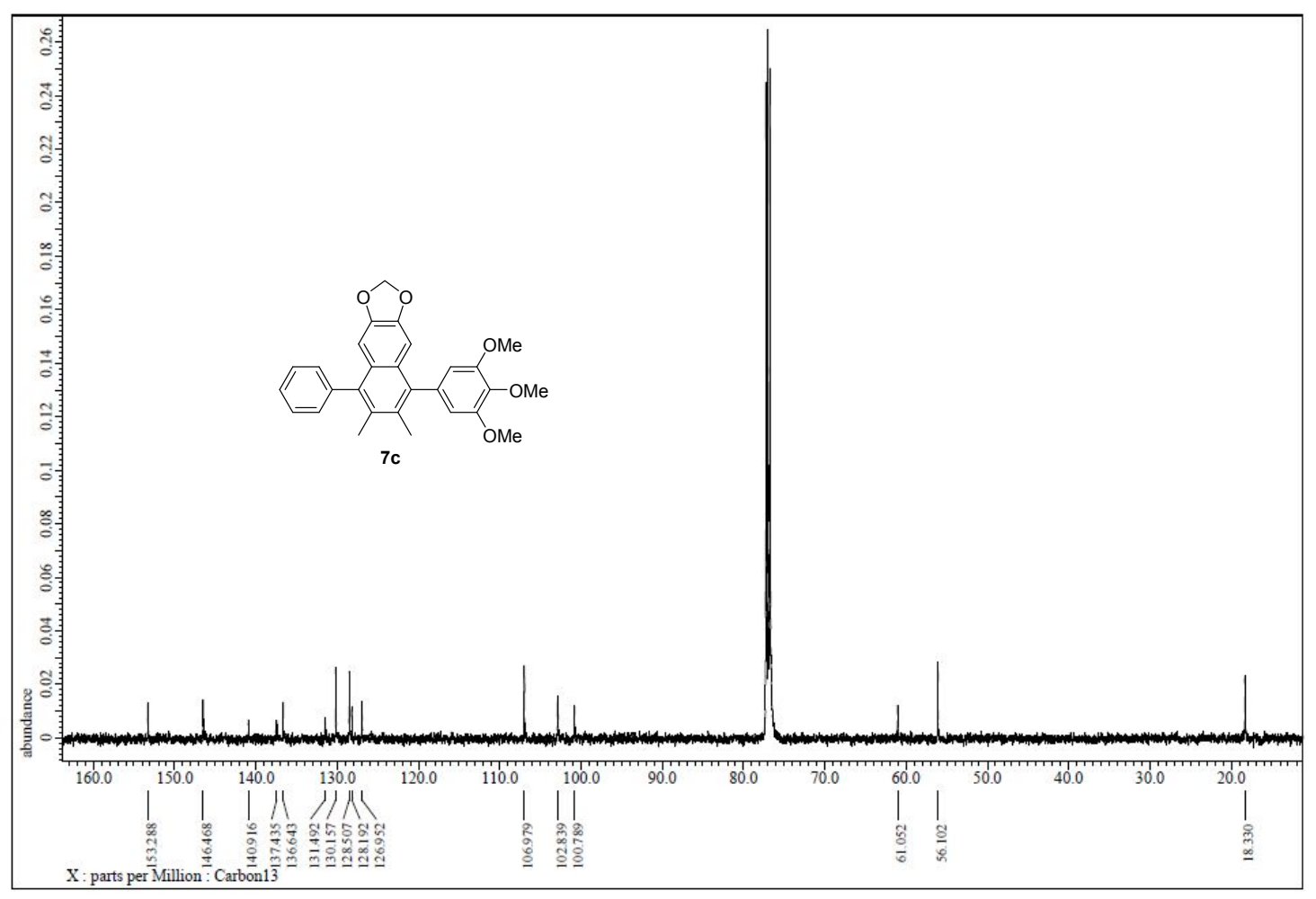

Figure S10: ${ }^{13} \mathrm{C}$ NMR (125 MHz, $\mathrm{CDCl}_{3}$ ) Spectrum of the Compound 7c 


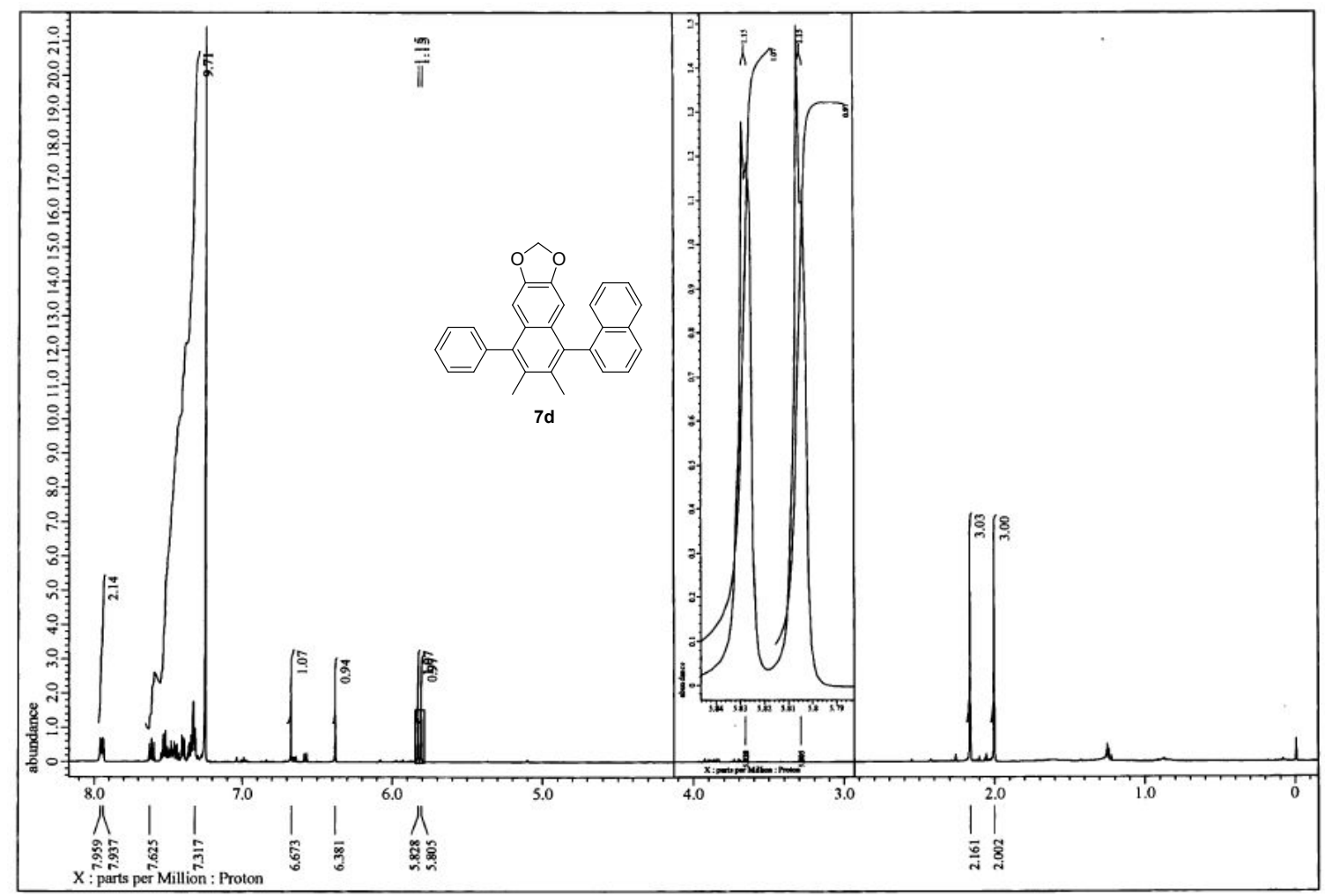

Figure S11: ${ }^{1} \mathrm{H}$ NMR (500 MHz, $\left.\mathrm{CDCl}_{3}\right)$ Spectrum of the Compound 7d



Figure S12: $\left.{ }^{13} \mathrm{C} \mathrm{NMR} \mathrm{(125} \mathrm{MHz}, \mathrm{CDCl}_{3}\right)$ Spectrum of the Compound 7d 


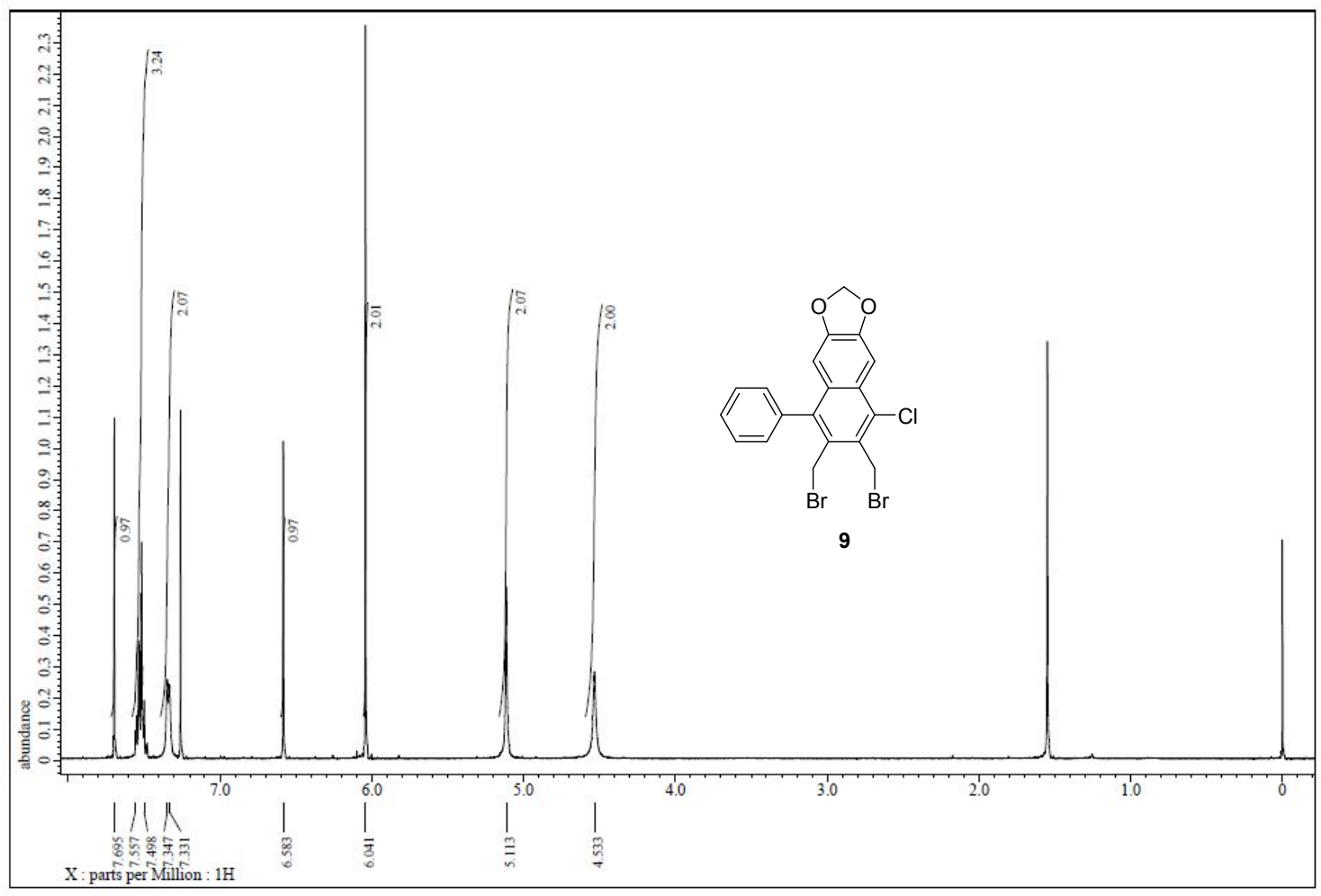

Figure S13: ${ }^{1} \mathrm{H}$ NMR (500 $\left.\mathrm{MHz}, \mathrm{CDCl}_{3}\right)$ Spectrum of the Compound 9

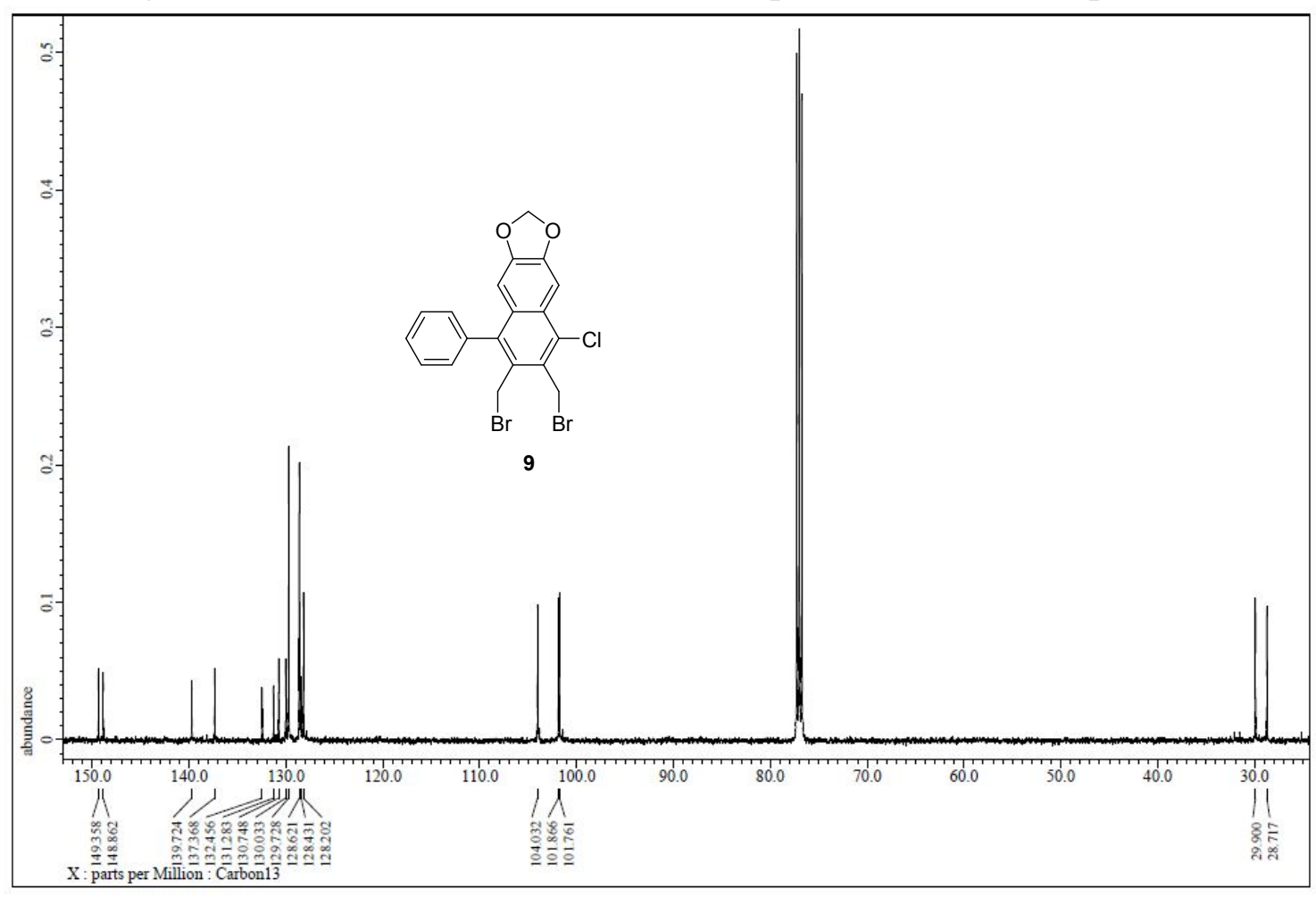

Figure S14: ${ }^{13} \mathrm{C}$ NMR (125 $\left.\mathrm{MHz}, \mathrm{CDCl}_{3}\right)$ Spectrum of the Compound 9 


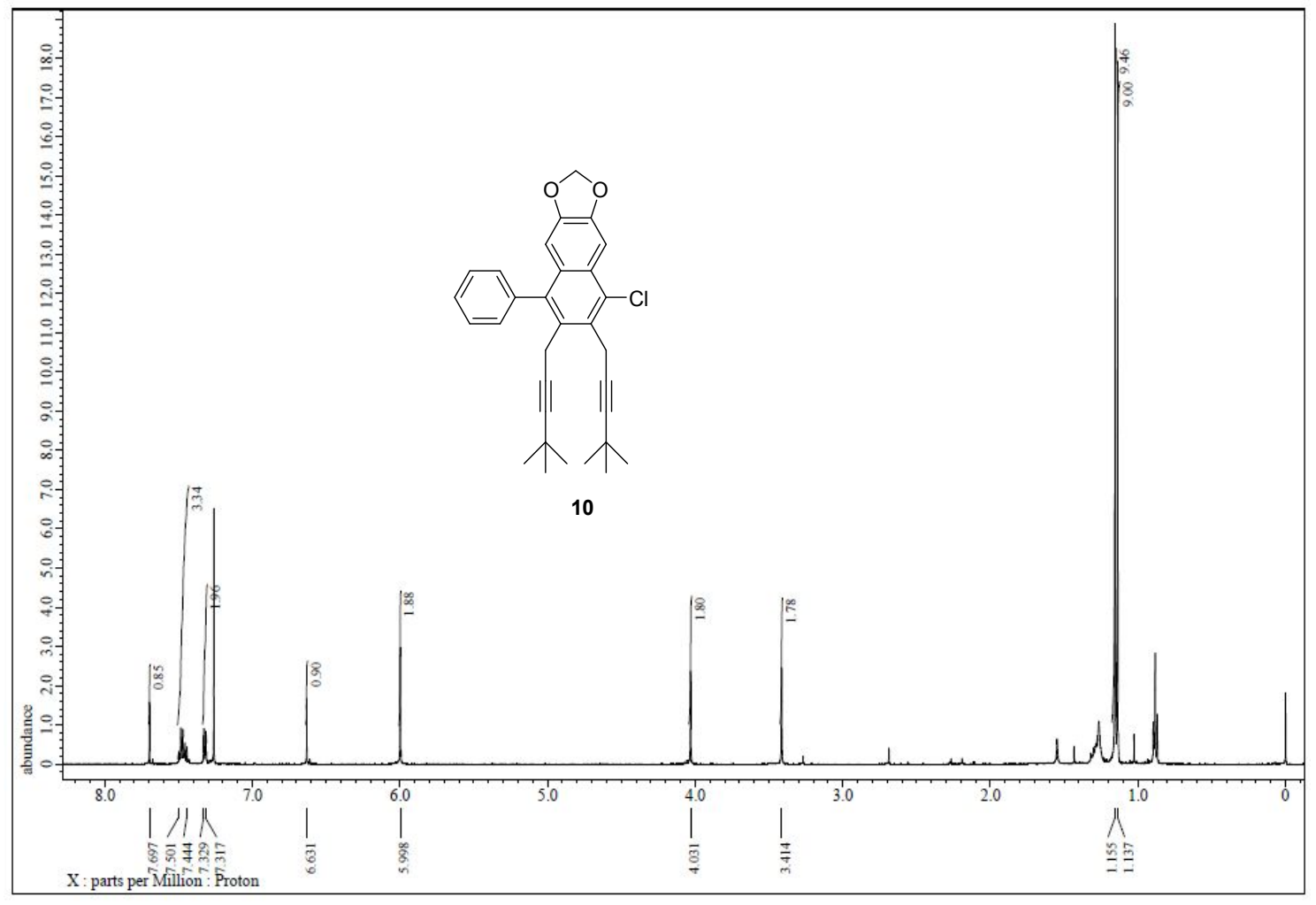

Figure S15: ${ }^{1} \mathrm{H}$ NMR (500 $\left.\mathrm{MHz} \mathrm{CDCl}_{3}\right)$ Spectrum of the Compound 10

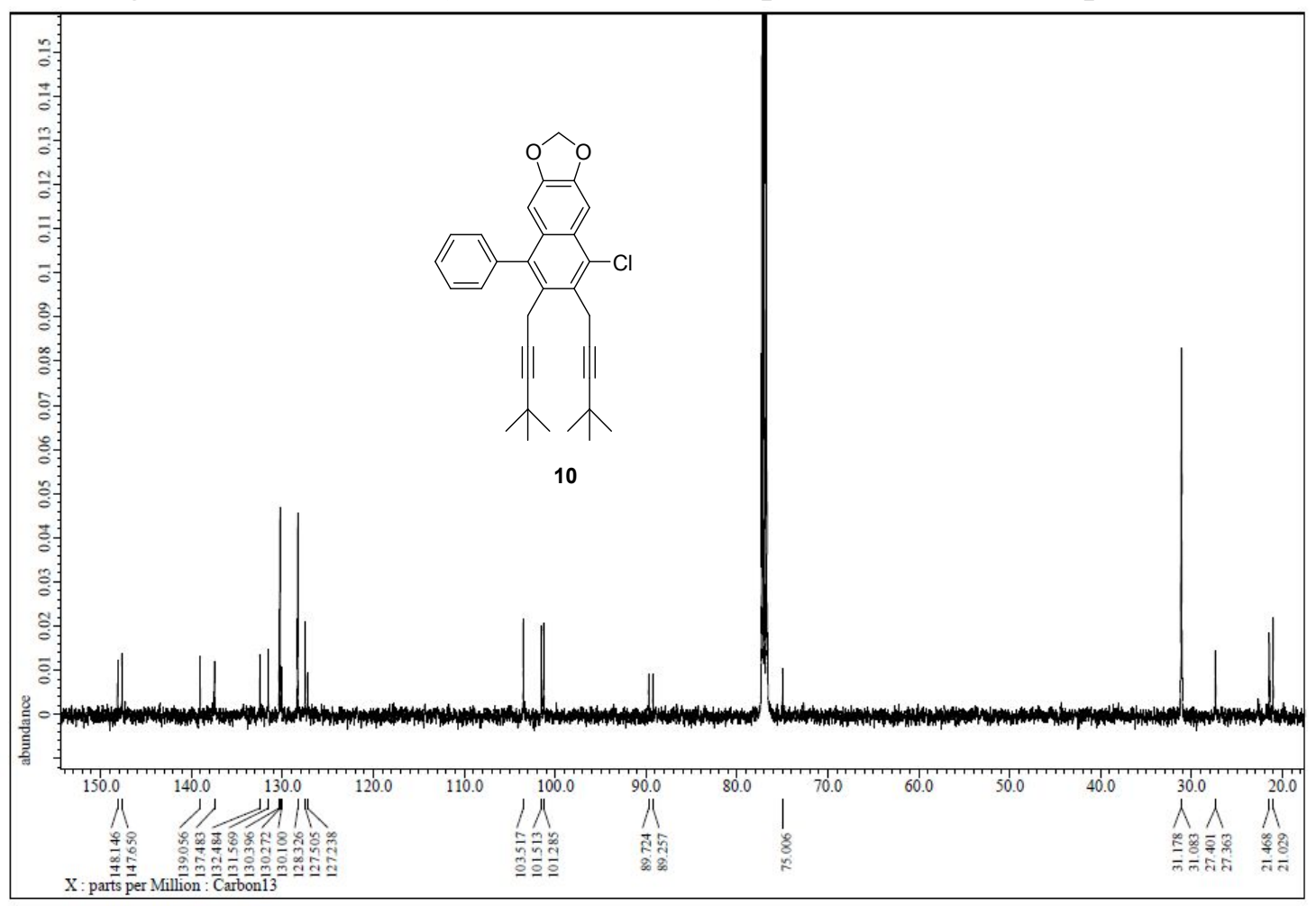

Figure S16: ${ }^{13} \mathrm{C}$ NMR (125 MHz, $\mathrm{CDCl}_{3}$ ) Spectrum of the Compound 10 


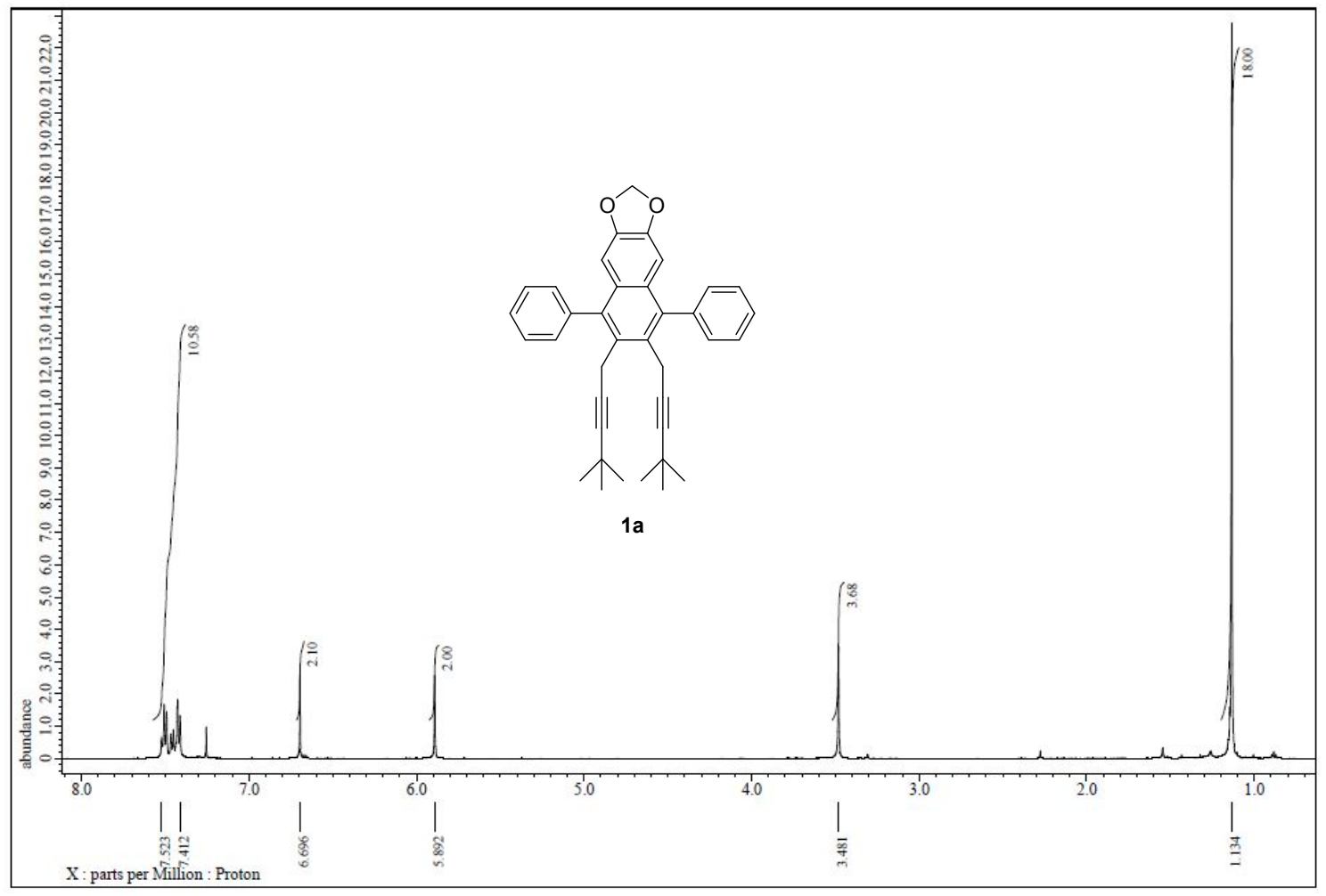

Figure S17: ${ }^{1} \mathrm{H}$ NMR (500 $\left.\mathrm{MHz}, \mathrm{CDCl}_{3}\right)$ Spectrum of the Compound 1a

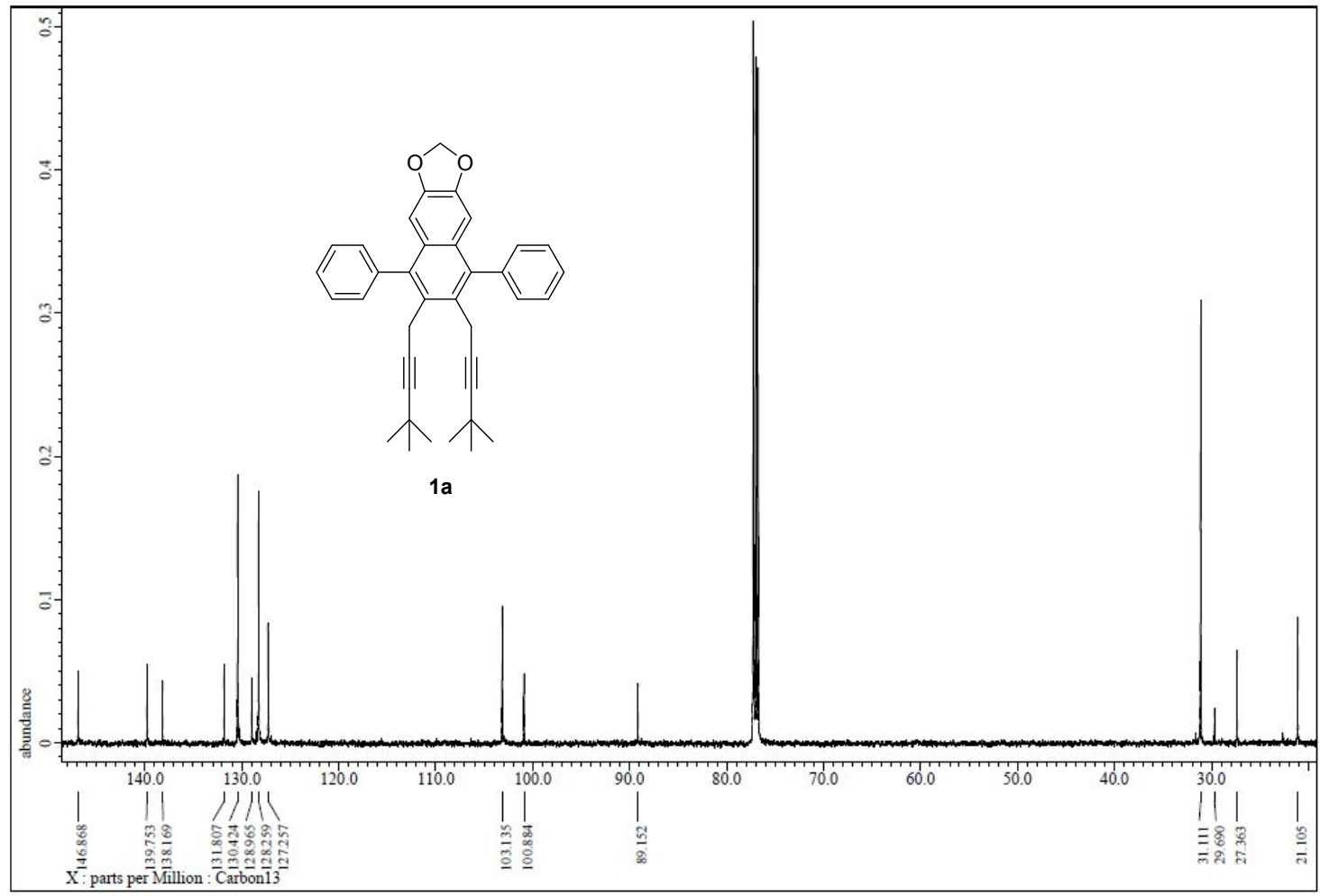

Figure S18: ${ }^{13} \mathrm{C}$ NMR (125 $\left.\mathrm{MHz}, \mathrm{CDCl}_{3}\right)$ Spectrum of the Compound 1a 


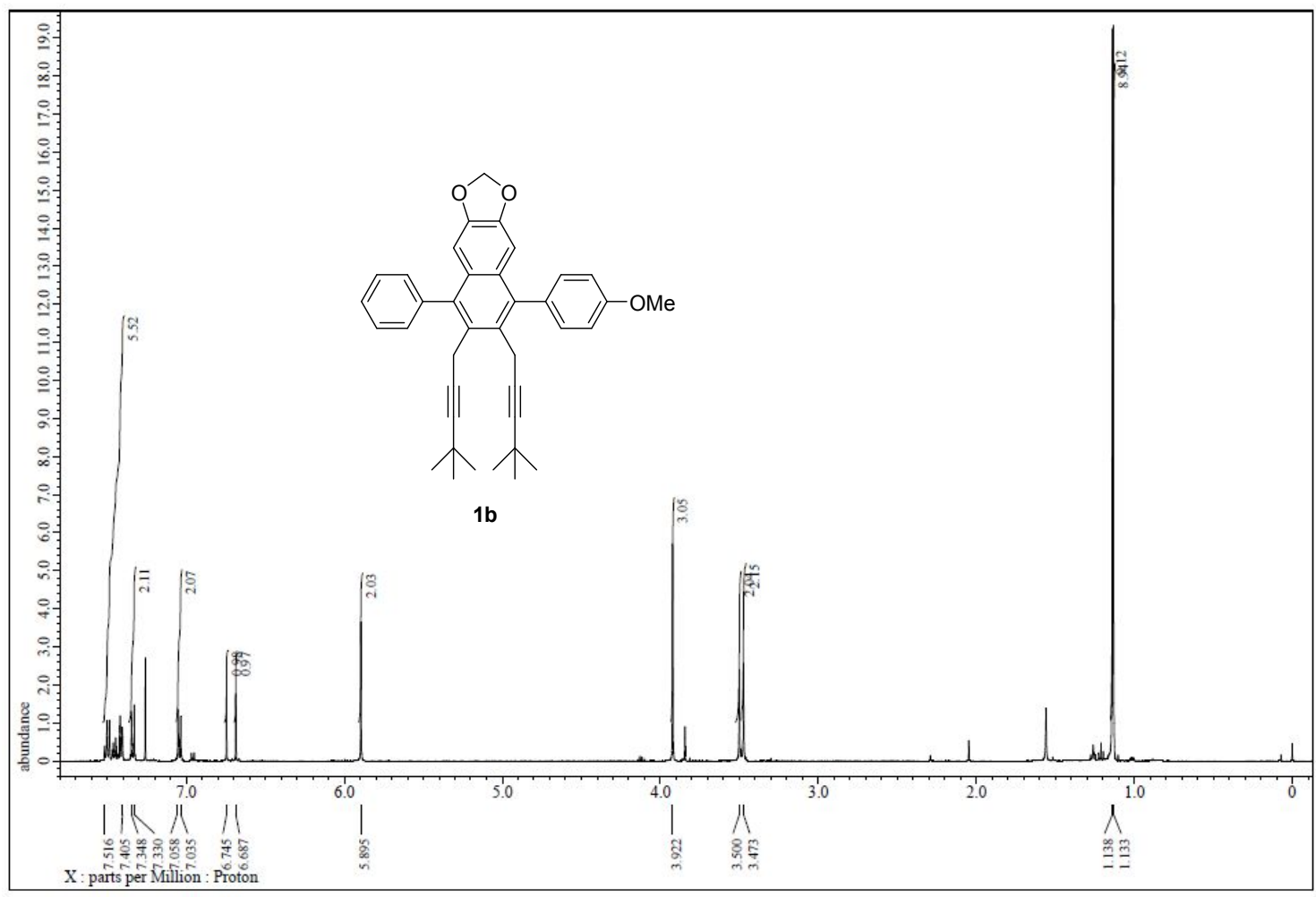

Figure S19: ${ }^{1} \mathrm{H}$ NMR (500 $\mathrm{MHz}, \mathrm{CDCl}_{3}$ ) Spectrum of the Compound $1 \mathrm{~b}$

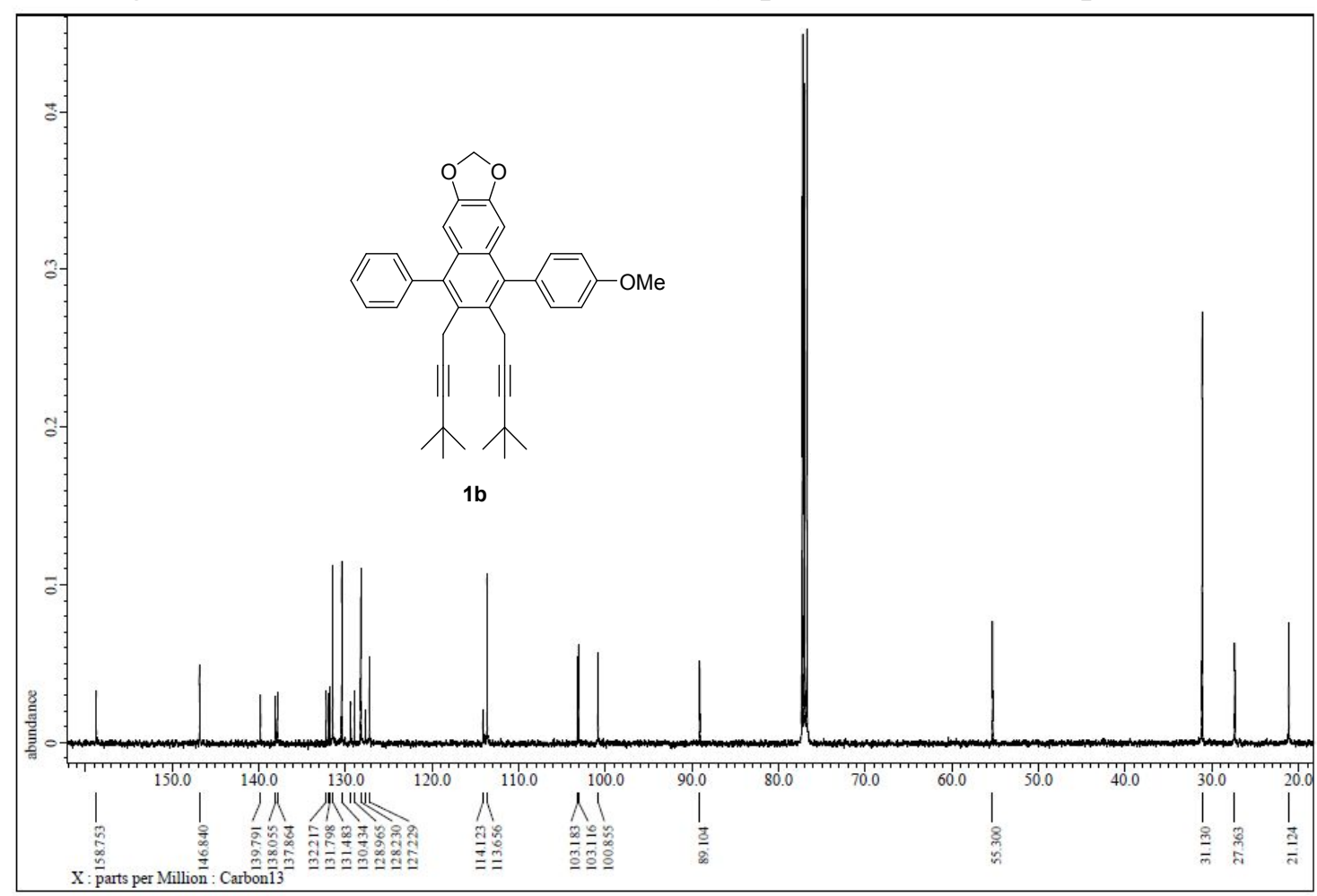

Figure S20: ${ }^{13} \mathrm{C}$ NMR (125 $\left.\mathrm{MHz}, \mathrm{CDCl}_{3}\right)$ Spectrum of the Compound $1 \mathrm{~b}$ 


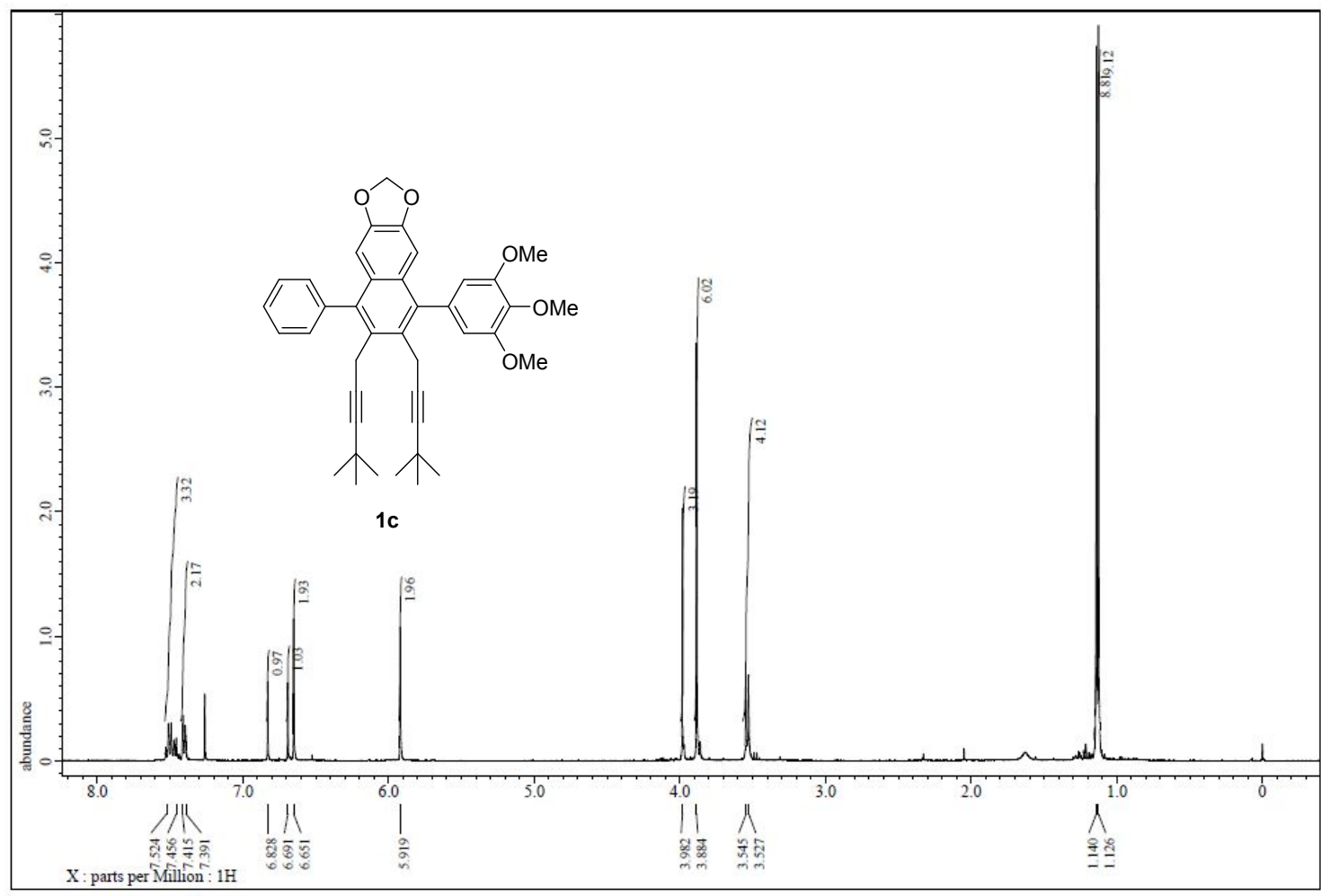

Figure S21: ${ }^{1} \mathrm{H}$ NMR (500 $\left.\mathrm{MHz} \mathrm{CDCl}_{3}\right)$ Spectrum of the Compound 1c

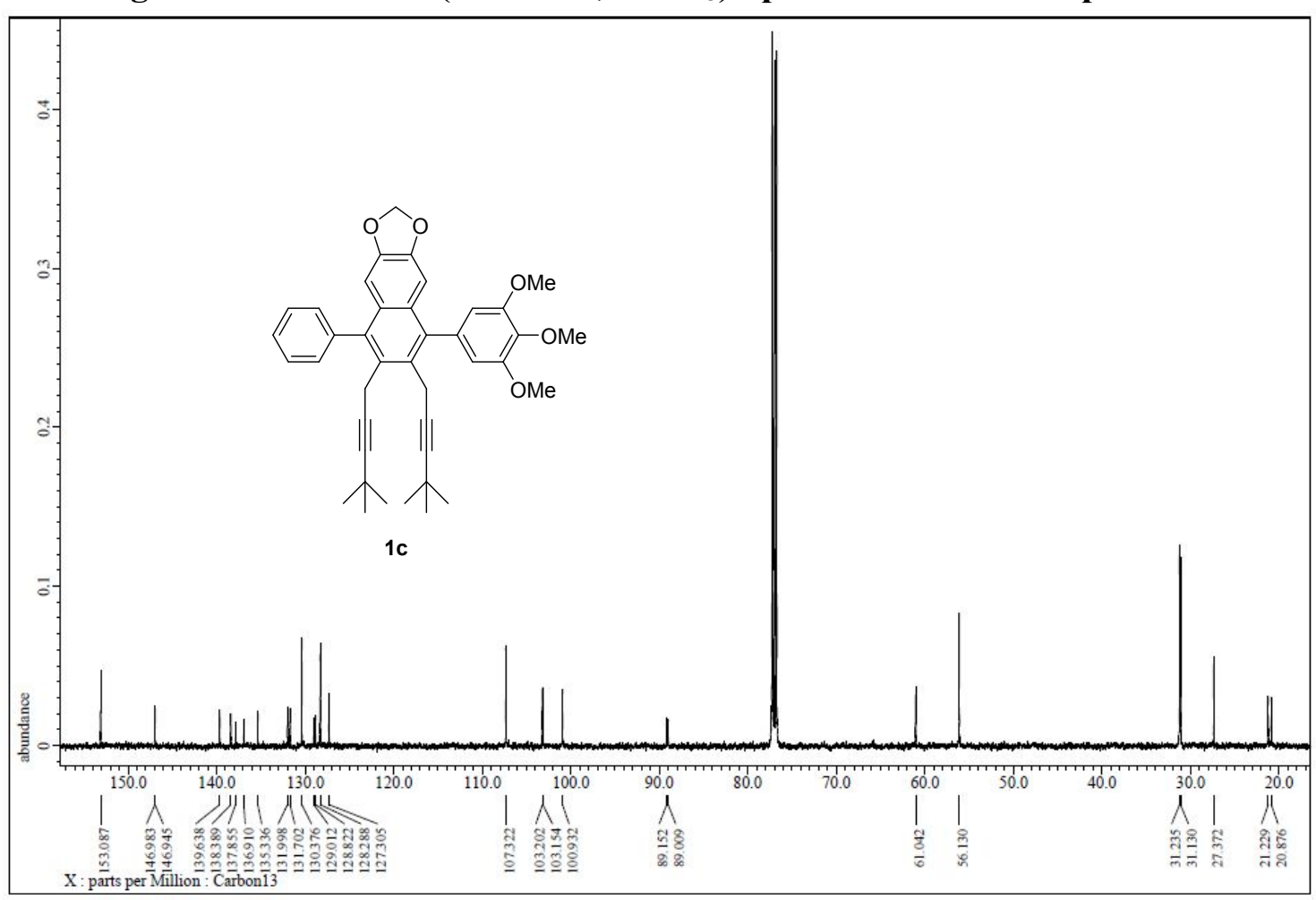

Figure S22: ${ }^{13} \mathrm{C}$ NMR (125 $\left.\mathrm{MHz}, \mathrm{CDCl}_{3}\right)$ Spectrum of the Compound 1c 


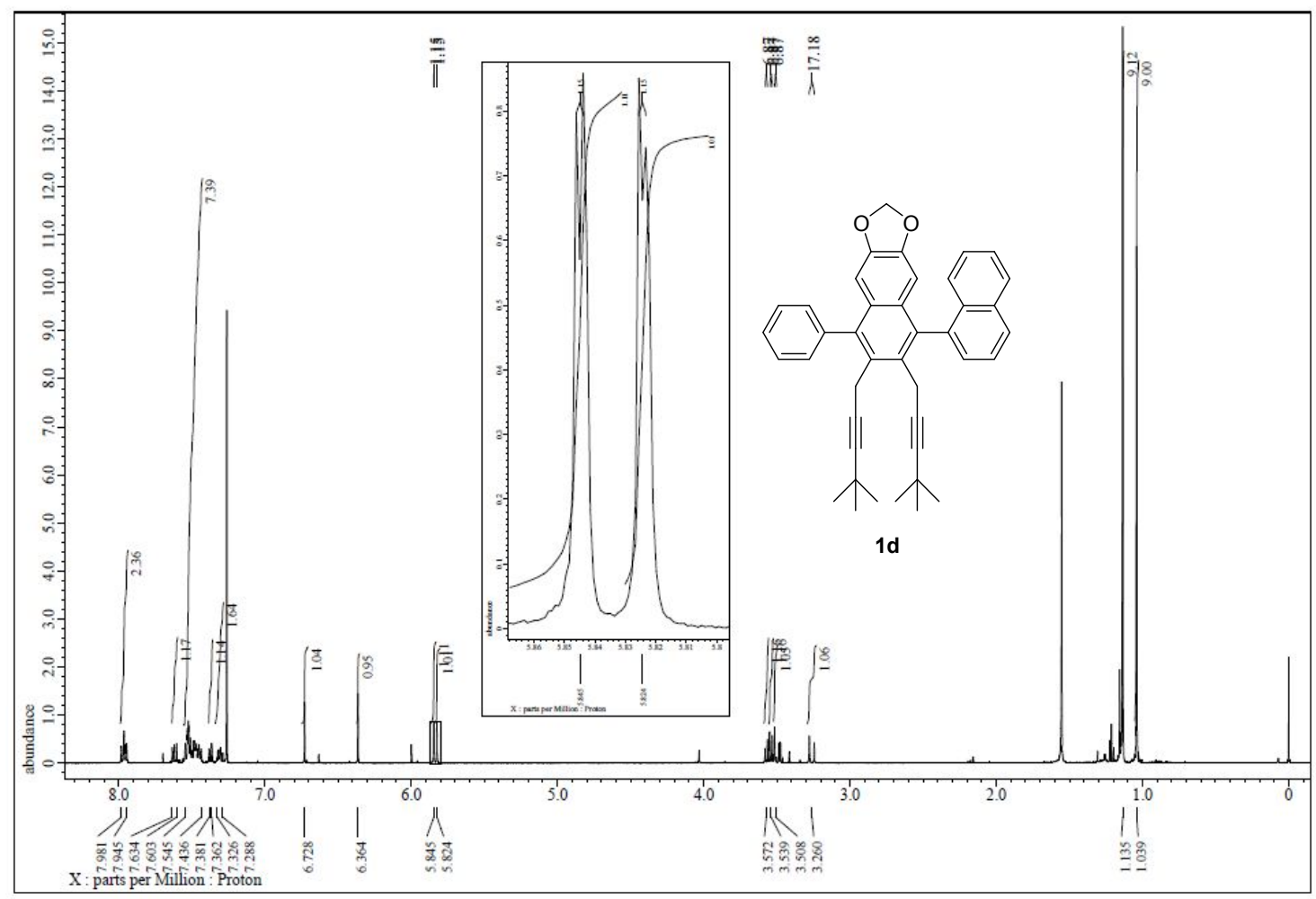

Figure S23: ${ }^{1} \mathrm{H}$ NMR (500 $\left.\mathrm{MHz}, \mathrm{CDCl}_{3}\right)$ Spectrum of the Compound 1d

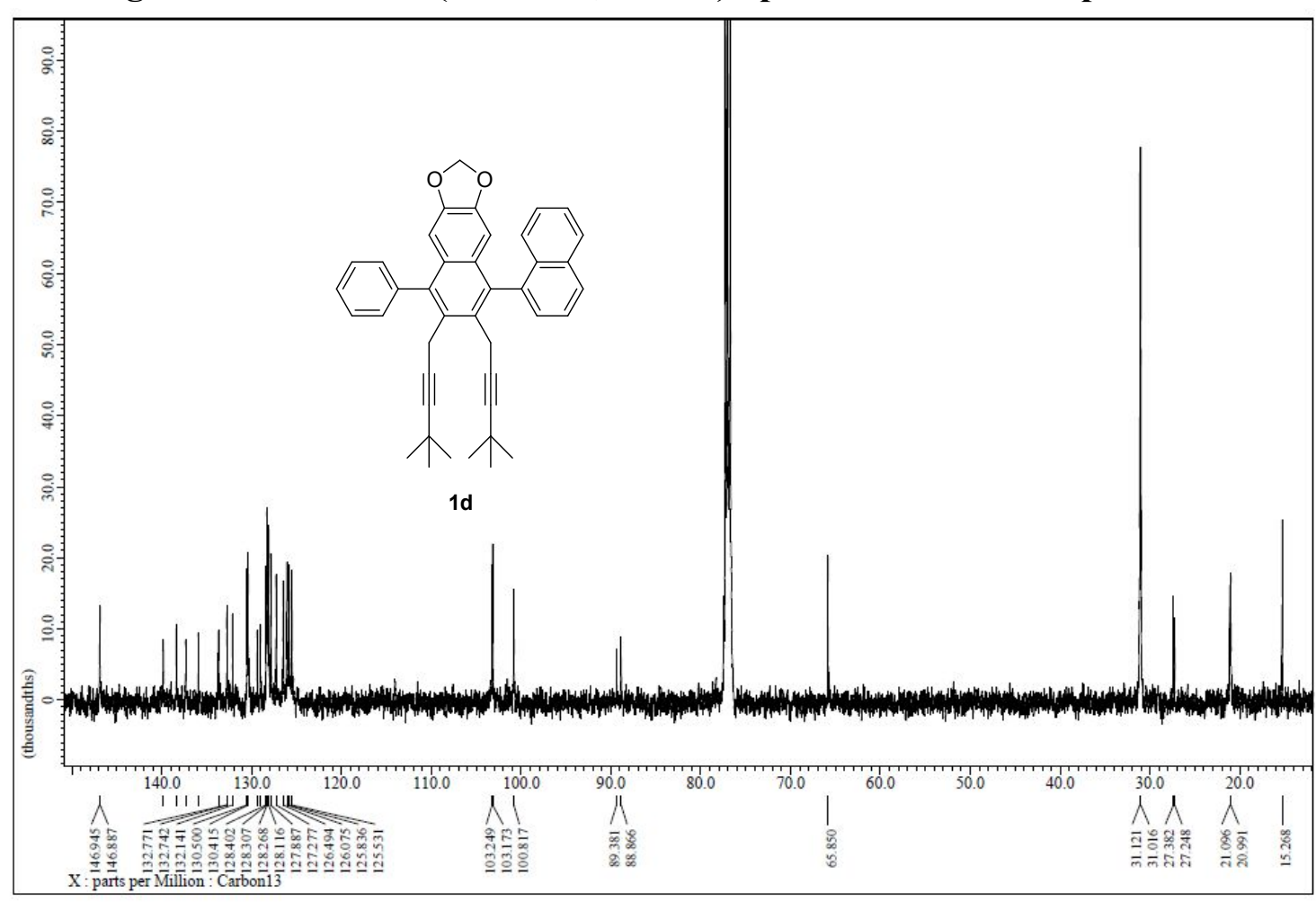

Figure S24: ${ }^{13} \mathrm{C}$ NMR (125 MHz, $\left.\mathrm{CDCl}_{3}\right)$ Spectrum of the Compound 1d 


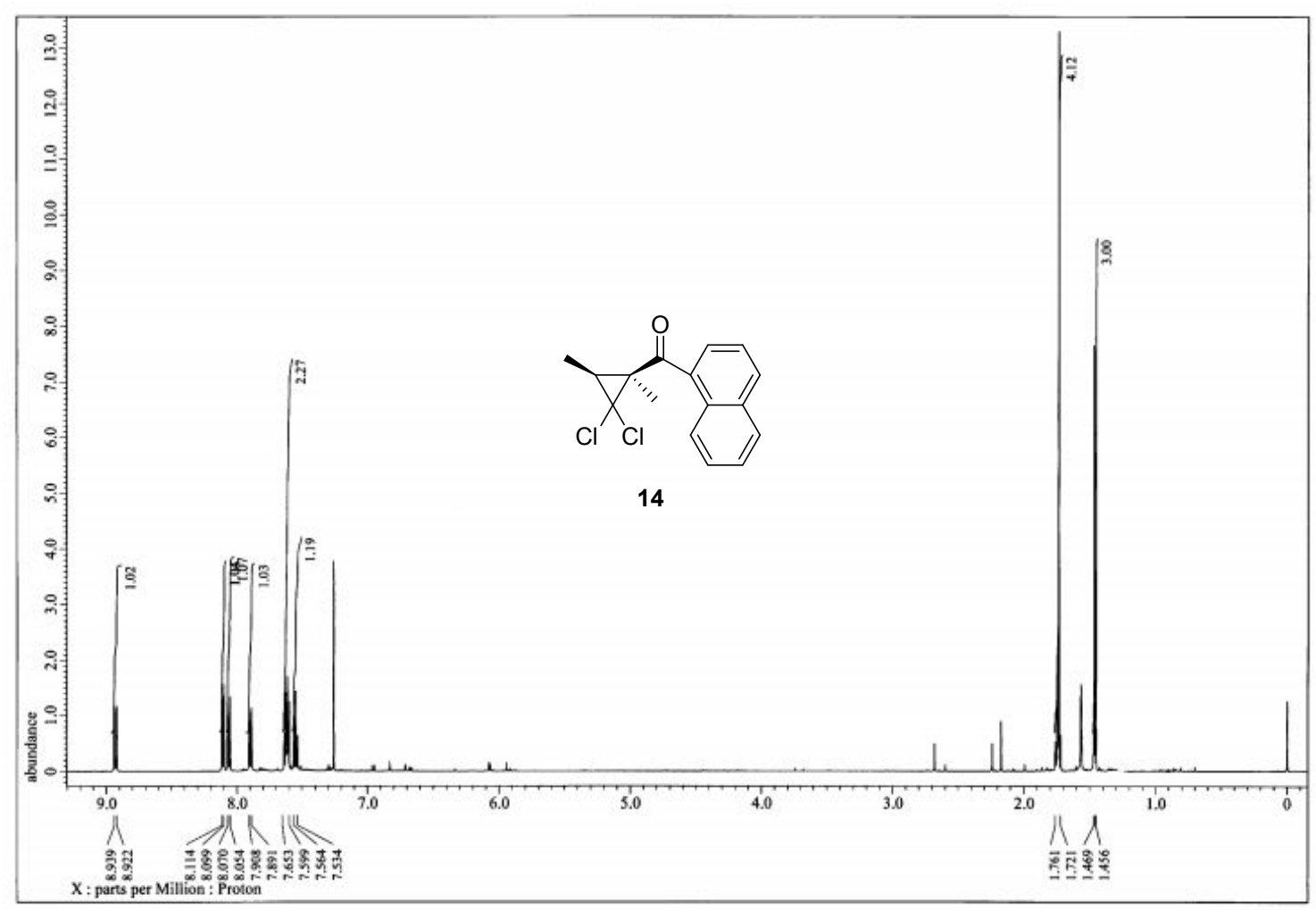

Figure S25: ${ }^{1} \mathrm{H}$ NMR (500 $\left.\mathrm{MHz}, \mathrm{CDCl}_{3}\right)$ Spectrum of the Compound 14

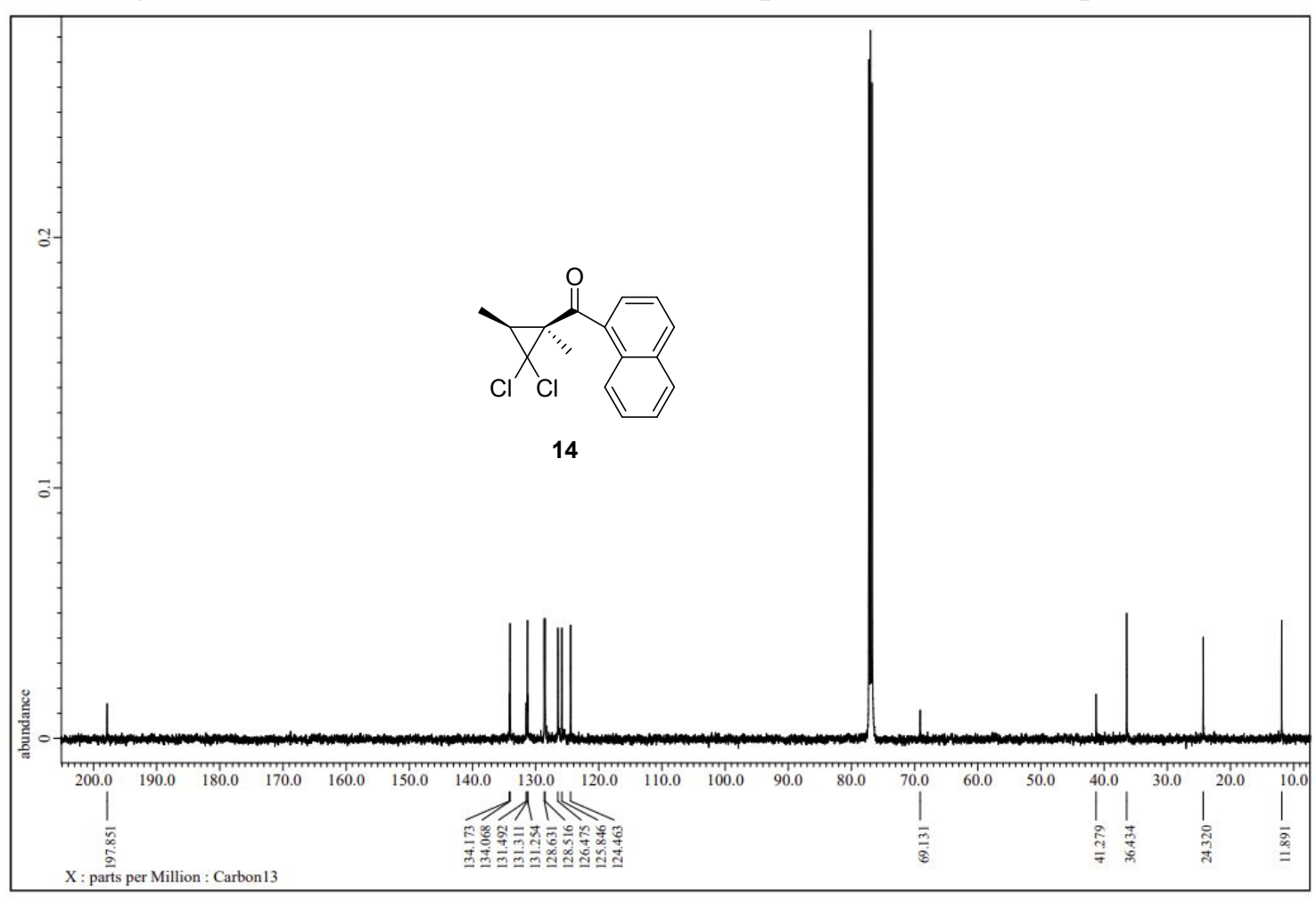

Figure S26: ${ }^{13} \mathrm{C}$ NMR (125 MHz, $\mathrm{CDCl}_{3}$ ) Spectrum of the Compound 14 


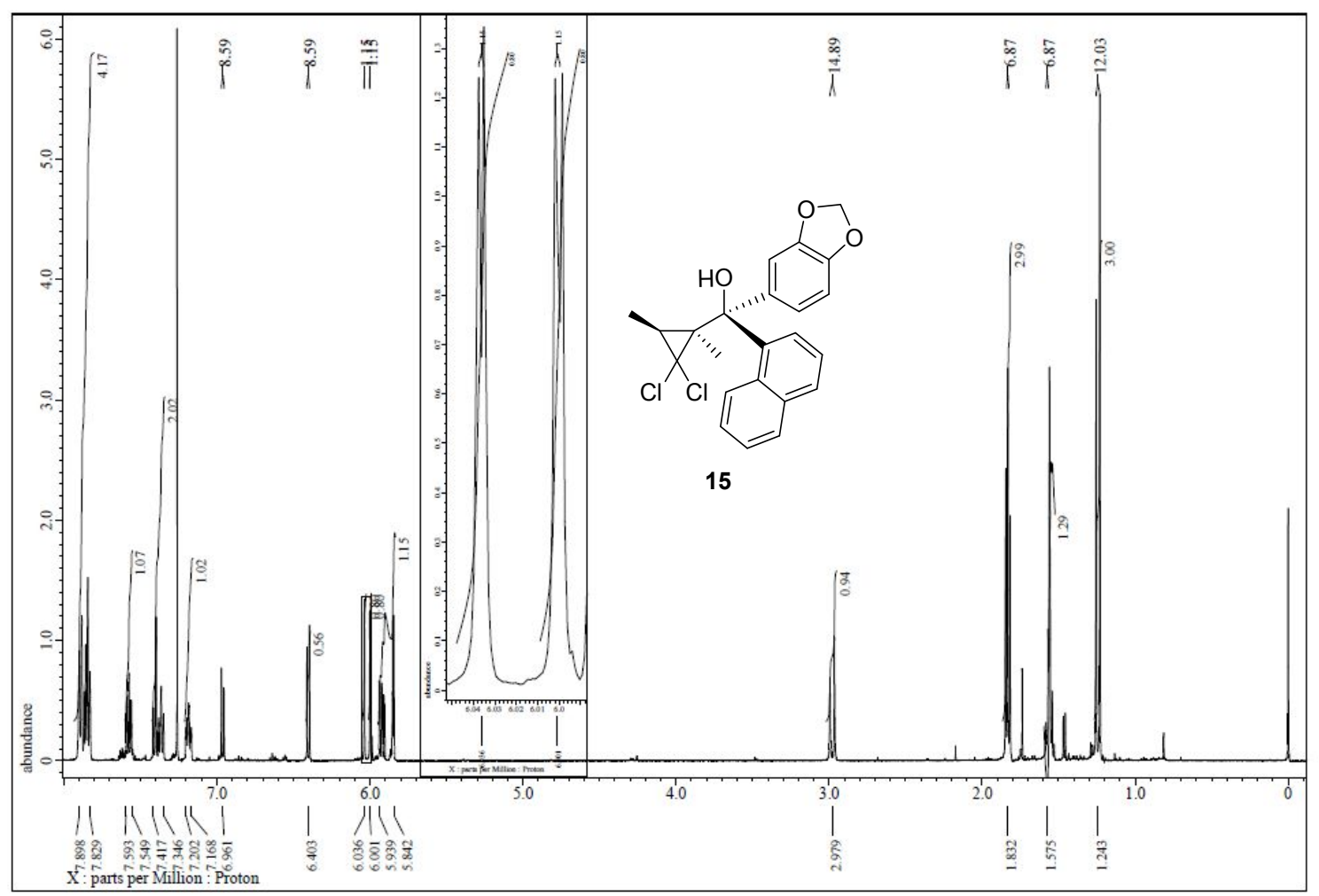

Figure S27: ${ }^{1} \mathrm{H}$ NMR (500 $\left.\mathrm{MHz} \mathrm{CDCl}_{3}\right)$ Spectrum of the Compound 15

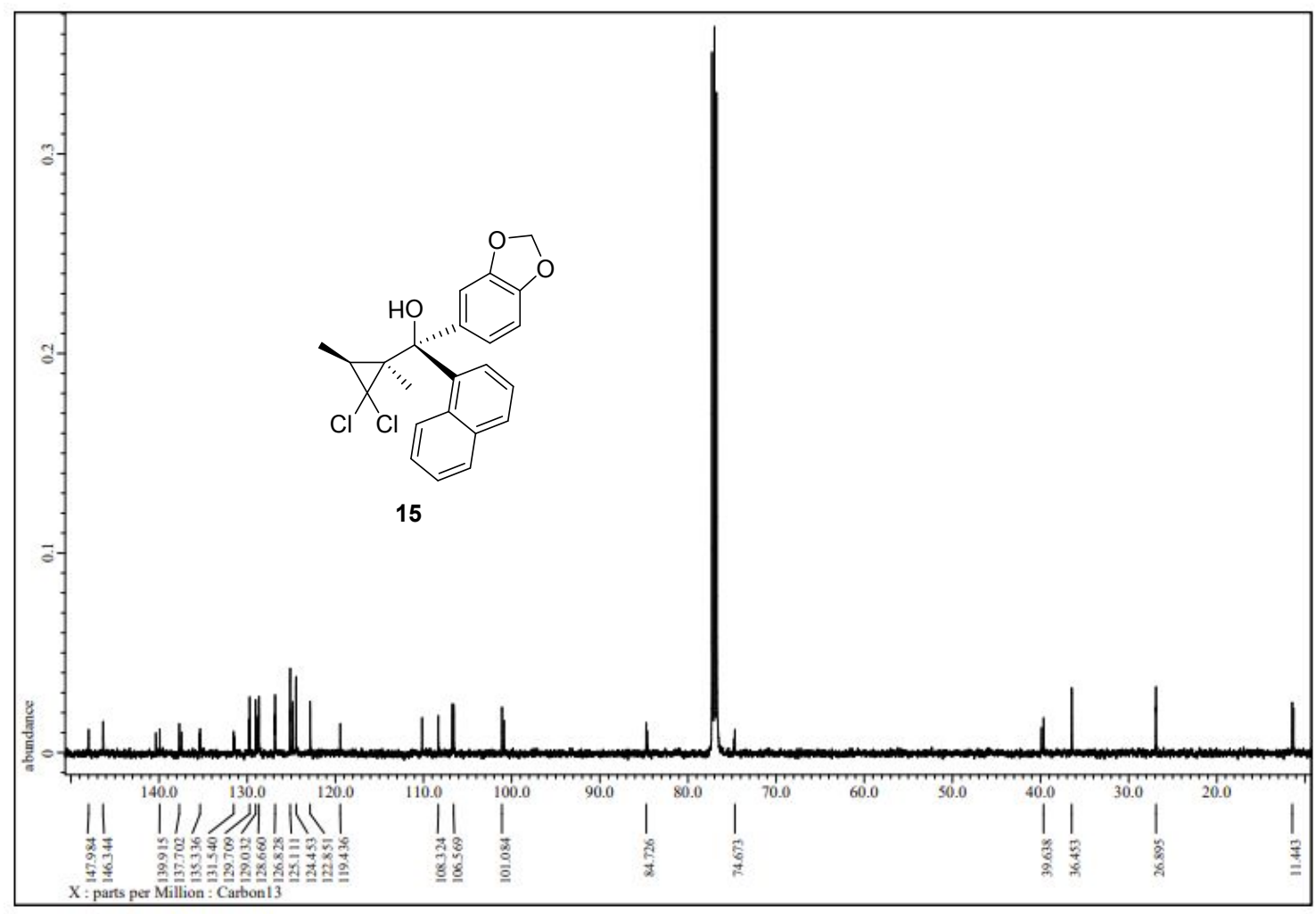

Figure S28: ${ }^{13} \mathrm{C}$ NMR (125 $\left.\mathrm{MHz}, \mathrm{CDCl}_{3}\right)$ Spectrum of the Compound 15 


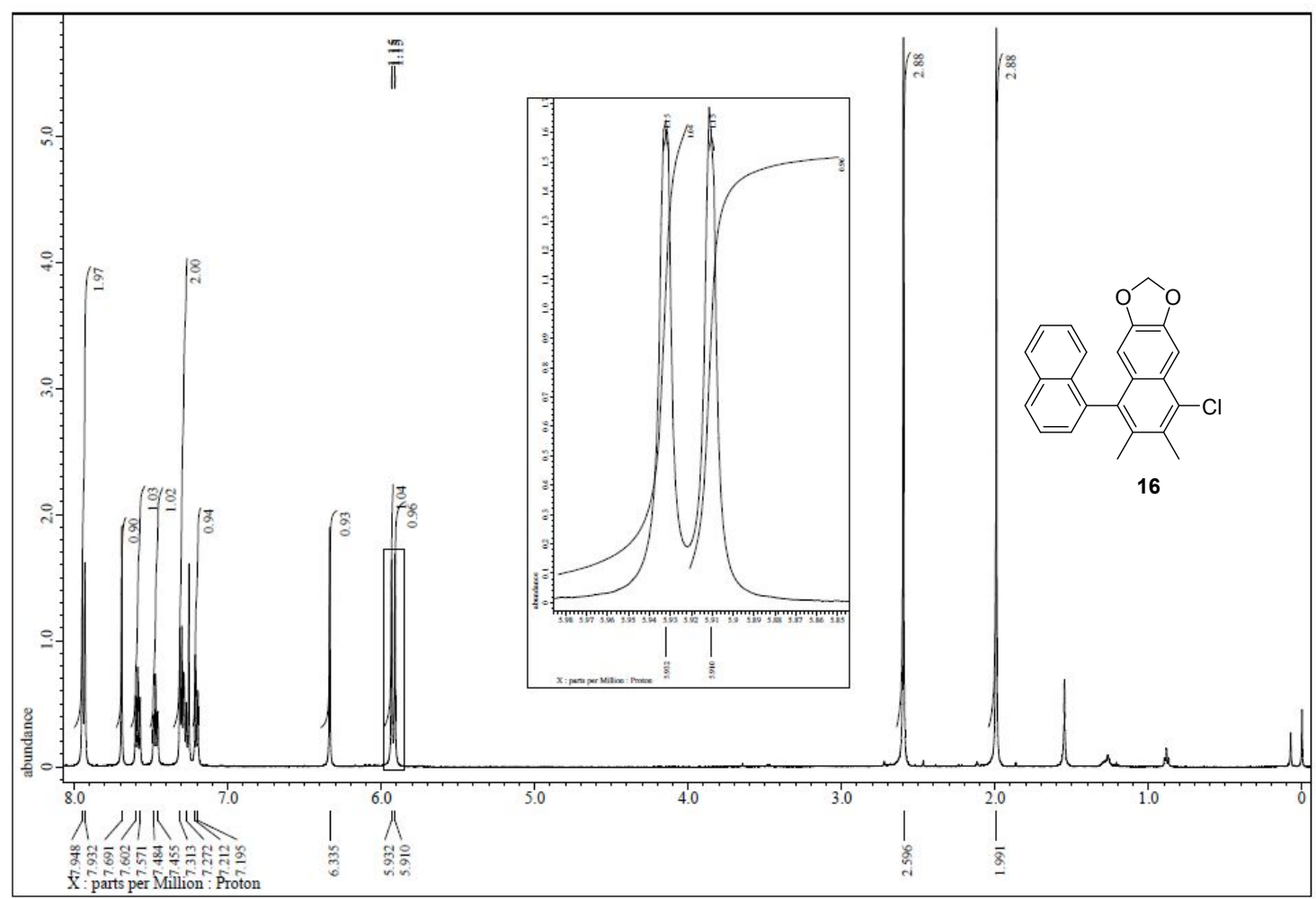

Figure S29: ${ }^{1} \mathrm{H}$ NMR (500 $\left.\mathrm{MHz} \mathrm{CDCl}_{3}\right)$ Spectrum of the Compound 16

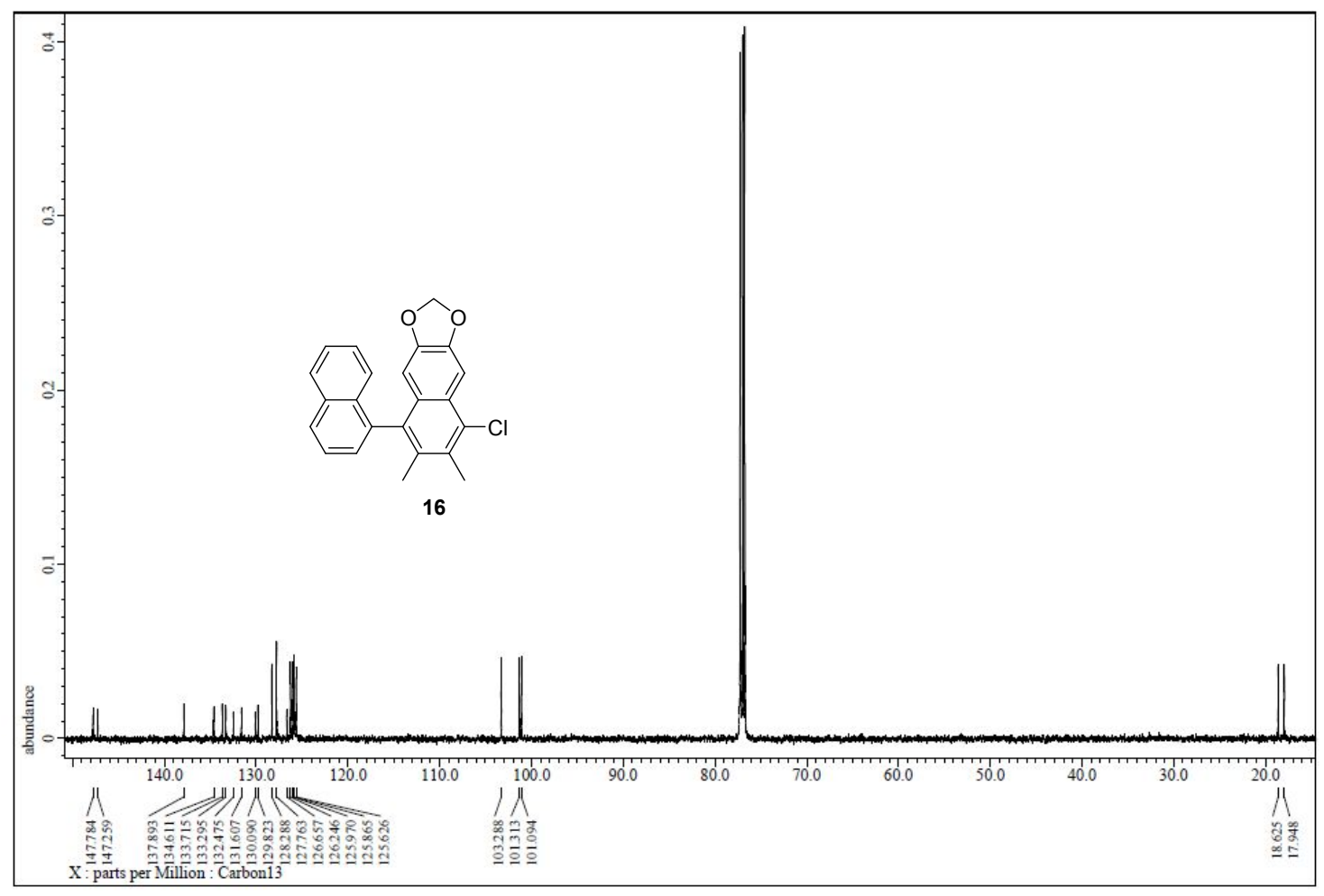

Figure S30: ${ }^{13} \mathrm{C}$ NMR (125 $\mathrm{MHz}, \mathrm{CDCl}_{3}$ ) Spectrum of the Compound 16 
$\mathrm{mV}$

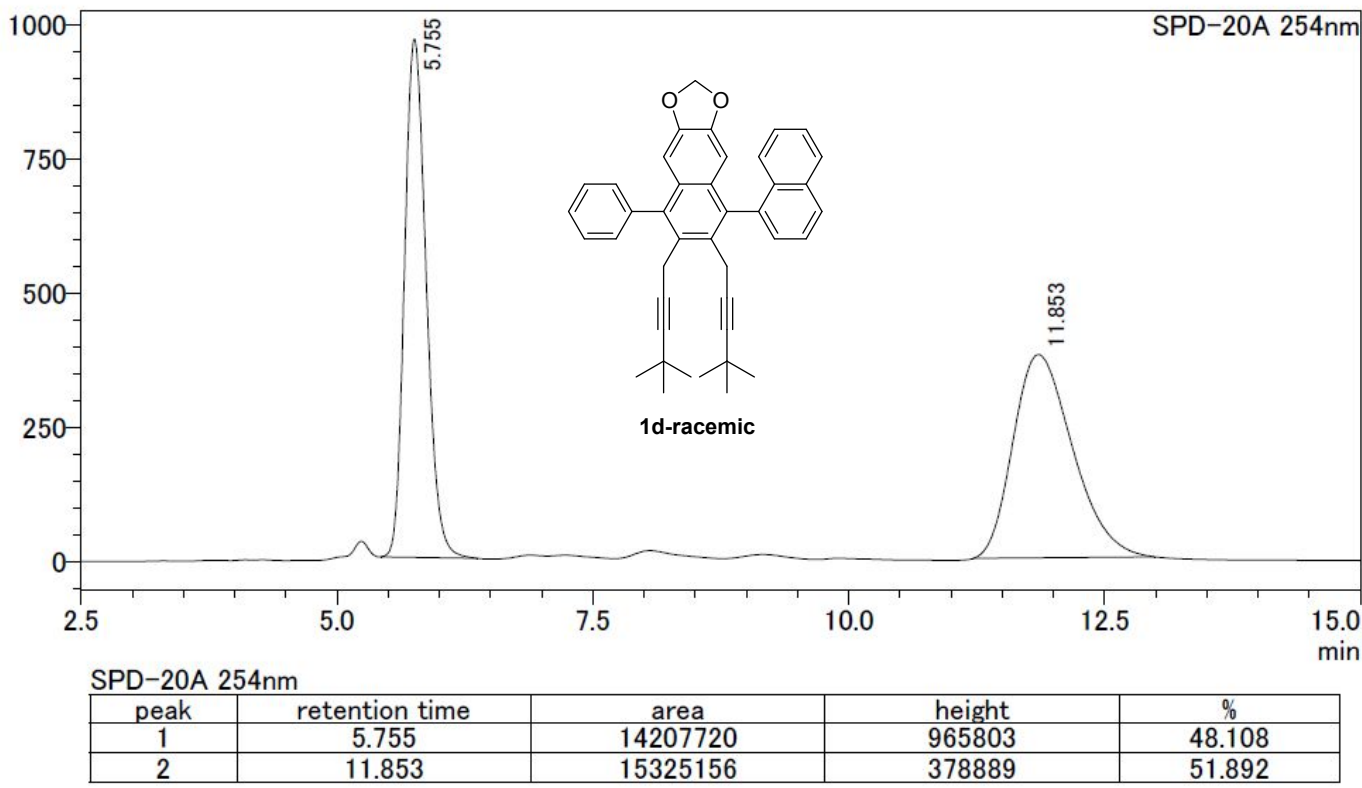

Figure S31: HPLC chart of the compound 1d (racemic) 
Table 1: Optimization of PEPPSI ${ }^{\mathrm{TM}}-\mathrm{IPr}-\mathrm{K}_{2} \mathrm{CO}_{3}$ catalysis using 6

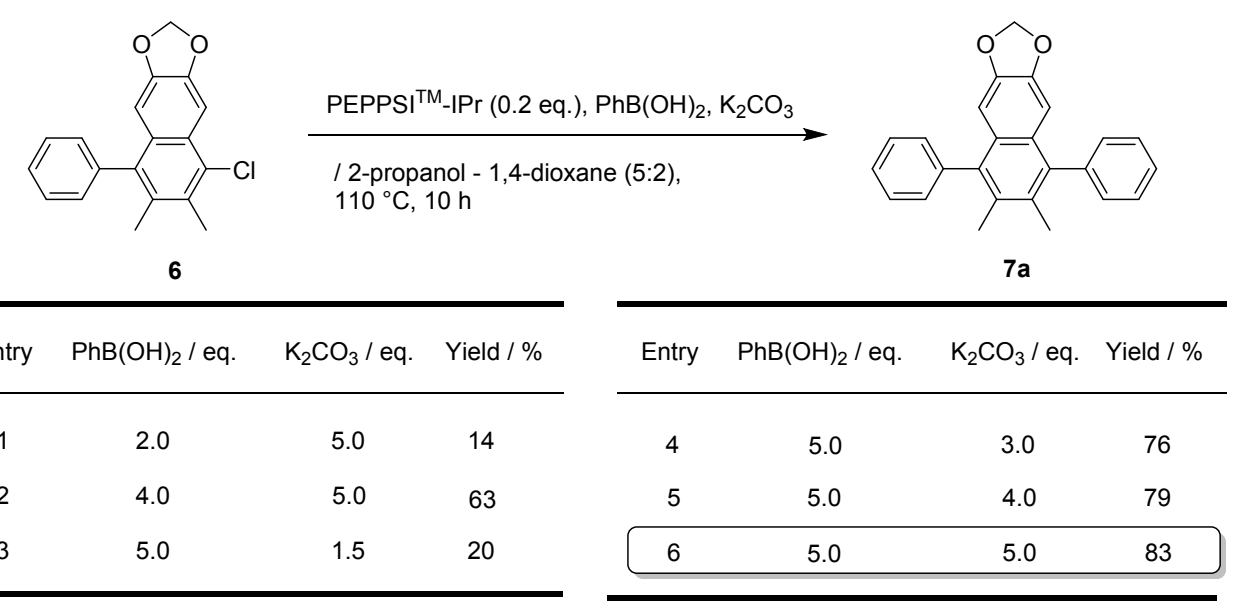


Data using Spartan software (Wavefunction, Inc. ver. '18 1.1.0) of 1a and 1d

\$comment

M0001

\$end

\$molecule

01

$6-1.1358911875-0.83203715223-2.1087001789$

$60.0349793710440 .71223068138-0.0085654726921$

$6-1.14249591390 .59665729347-2.1837267412$

$6-0.56185037549-1.4773538199-0.97187417094$

$60.0054552879333-0.707451248360 .085137473634$

$6-0.554818664941 .3622929979-1.1316898837$

$6-1.73834954951 .2151189353-3.3168607506$

$6-2.28279106450 .44529116106-4.3189867007$

$6-2.2666729804-0.91591131582-4.2527153386$

$6-1.7094550543-1.5716268733-3.1790078947$

$1-1.78000003422 .2950739601-3.4241126747$

$1-1.7256349132-2.6575718231-3.1807140203$

$8-2.847530896-1.4995714873-5.3337678237$

$6-3.2332371647-0.33459396269-6.0919552568$

$8-2.87630736230 .90730691725-5.4508040255$

$1-4.3193794181-0.35469474091-6.238292717$

$1-2.7451617005-0.37644171549-7.0725445374$

$60.61292888046-1.41549031931 .2948723254$

$10.083724244916-2.34969217491 .5102122816$

$10.45408805545-0.826272605152 .2039035671$

60.66144689251 .54802400821 .1062349318

11.09379168552 .47236764250 .70896324359

11.5287143121 .03222448681 .5311527943

$62.0481510761-1.68439472521 .110038672$

$6-0.308993052671 .86471671112 .1665720538$

$63.2159609827-1.9112673210 .94061078886$

$6-1.11447492732 .12877194623 .0182811267$

$64.6486245551-2.19904304750 .70853600992$

$6-2.12286573552 .46000665044 .0491371439$

$65.2845202089-2.70705038952 .0095614666$

$16.3483364361-2.93027371831 .8703462454$

$14.794216263-3.62226248242 .3624146321$

$15.200962094-1.96354135812 .8112540065$

$65.3578921286-0.915633543360 .25497779281$

$14.920741875-0.52439875308-0.67154926889$

$16.4234810298-1.09461139930 .072308029566$

$64.7792992403-3.2719423466-0.38187440951$

$14.3285887105-2.9394473567-1.324724084$

$14.2741522754-4.2006732656-0.090424662568$

$15.8303576082-3.510690565-0.57963602075$

$6-1.42705432283 .10583428615 .2549029565$

$1-0.68337447572 .430762365 .6950370756$

$1-2.14885709713 .36408533656 .037987521$

$1-0.902304416134 .02485786524 .9675876514$

$6-2.83682571121 .17579264784 .4929518298$

$1-3.59348403881 .38638768775 .2571510434$

$1-2.12934886640 .451046596654 .9133865989$

$6-3.14670261683 .4403151463 .459287263$ 
$1-3.9104775823 .7084345954 .1979325435$

$1-3.65825320893 .00824422632 .5907899479$

$1-2.66499374844 .36685645413 .1244230226$

$15.2761240422-0.126047293231 .0115574336$

$1-3.33986265120 .687281563753 .6497873452$

$6-0.55639827061-2.9564142068-0.89156934211$

$6-0.54697740683-5.7623283957-0.74380071176$

$6-1.6609086882-3.6507199614-0.3708792168$

$60.55022530882-3.6943488976-1.3409638684$

$60.55564307161-5.088292306-1.2652847763$

$6-1.6554401964-5.0450185571-0.29752079476$

$1-2.5324887195-3.1013404111-0.020898405376$

$11.4174423135-3.1788299263-1.749023413$

$11.4211858617-5.6459375994-1.612906792$

$1-2.5163852911-5.57021180160 .10753787714$

$1-0.5424383159-6.8476555777-0.68561757801$

$6-0.55863849342 .8415122542-1.2090676199$

$6-0.569121414375 .6472979904-1.3591619355$

$60.460124238023 .5268745327-1.8913667158$

$6-1.58464120013 .5878483915-0.60788996537$

$6-1.58838777394 .9819569221-0.68058716914$

$60.454414351474 .9211388127-1.9653414456$

$11.26349860582 .9703873248-2.3697693889$

$1-2.38523512243 .0792815206-0.074272151241$

$1-2.38679065655 .5466013359-0.20655837572$

$11.24857467235 .4393652083-2.4962635013$

$1-0.572837745436 .7327042015-1.4159141218$

\$end

Srem

JOBTYPE OPT

TIDY_SYM TRUE

EXCHANGE B3LYP

CORRELATION none (built-in)

BASIS 6-31G(d)

VARTHRESH 2 (default DFT)

INCDFT TRUE (default DFT)

GUI GUI_SPARTAN

TERSE_OUTPUT TRUE

Send

\$opt

\$end

Standard Nuclear Orientation (Angstroms)

I Atom X Y Z

1 C $-1.1358911875-0.8320371522-2.1087001789$

2 C $0.03497937100 .7122306814-0.0085654727$

3 C $-1.14249591390 .5966572935-2.1837267412$

4 C $-0.5618503755-1.4773538199-0.9718741709$

5 C $0.0054552879-0.70745124840 .0851374736$

6 C $-0.55481866491 .3622929979-1.1316898837$

7 C $-1.73834954951 .2151189353-3.3168607506$

8 C $-2.28279106450 .4452911611-4.3189867007$

9 C $-2.2666729804-0.9159113158-4.2527153386$

10 C $-1.7094550543-1.5716268733-3.1790078947$ 
$11 \mathrm{H}-1.78000003422 .2950739601-3.4241126747$

$12 \mathrm{H}-1.7256349132-2.6575718231-3.1807140203$

13 O $-2.8475308960-1.4995714873-5.3337678237$

14 C $-3.2332371647-0.3345939627-6.0919552568$ 15 O $-2.87630736230 .9073069173-5.4508040255$

$16 \mathrm{H}-4.3193794181-0.3546947409-6.2382927170$

$17 \mathrm{H}-2.7451617005-0.3764417155-7.0725445374$

18 C $0.6129288805-1.41549031931 .2948723254$

19 H $0.0837242449-2.34969217491 .5102122816$

20 H $0.4540880555-0.82627260522 .2039035671$

$21 \mathrm{C} 0.66144689251 .54802400821 .1062349318$

22 H 1.09379168552 .47236764250 .7089632436

23 H 1.52871431201 .03222448681 .5311527943

24 C $2.0481510761-1.68439472521 .1100386720$

25 C -0.30899305271 .86471671112 .1665720538$

26 C $3.2159609827-1.91126732100 .9406107889$

27 C -1.11447492732 .12877194623 .0182811267$

28 C $4.6486245551-2.19904304750 .7085360099$

29 C -2.12286573552 .46000665044 .0491371439$

30 C $5.2845202089-2.70705038952 .0095614666$

31 H $6.3483364361-2.93027371831 .8703462454$

32 H $4.7942162630-3.62226248242 .3624146321$

33 H $5.2009620940-1.96354135812 .8112540065$

34 C $5.3578921286-0.91563354340 .2549777928$

35 H $4.9207418750-0.5243987531-0.6715492689$

36 H $6.4234810298-1.09461139930 .0723080296$

37 C $4.7792992403-3.2719423466-0.3818744095$

38 H $4.3285887105-2.9394473567-1.3247240840$

39 H $4.2741522754-4.2006732656-0.0904246626$

40 H $5.8303576082-3.5106905650-0.5796360207$

$41 \mathrm{C}-1.42705432283 .10583428615 .2549029565$

$42 \mathrm{H}-0.68337447572 .43076236005 .6950370756$

$43 \mathrm{H}-2.14885709713 .36408533656 .0379875210$

$44 \mathrm{H}-0.90230441614 .02485786524 .9675876514$

45 C -2.83682571121 .17579264784 .4929518298$

$46 \mathrm{H}-3.59348403881 .38638768775 .2571510434$

$47 \mathrm{H}-2.12934886640 .45104659674 .9133865989$

48 C -3.14670261683 .44031514603 .4592872630$

49 H -3.91047758203 .70843459504 .1979325435$

$50 \mathrm{H}-3.65825320893 .00824422632 .5907899479$

$51 \mathrm{H}-2.66499374844 .36685645413 .1244230226$

52 H $5.2761240422-0.12604729321 .0115574336$

$53 \mathrm{H}-3.33986265120 .68728156383 .6497873452$

$54 \mathrm{C}-0.5563982706-2.9564142068-0.8915693421$ 55 C $-0.5469774068-5.7623283957-0.7438007118$ 56 C $-1.6609086882-3.6507199614-0.3708792168$ 57 C $0.5502253088-3.6943488976-1.3409638684$ 58 C $0.5556430716-5.0882923060-1.2652847763$ 59 C $-1.6554401964-5.0450185571-0.2975207948$ $60 \mathrm{H}-2.5324887195-3.1013404111-0.0208984054$ 61 H $1.4174423135-3.1788299263-1.7490234130$ 62 H $1.4211858617-5.6459375994-1.6129067920$ $63 \mathrm{H}-2.5163852911-5.57021180160 .1075378771$ $64 \mathrm{H}-0.5424383159-6.8476555777-0.6856175780$ 65 C $-0.55863849342 .8415122542-1.2090676199$ 66 C $-0.56912141445 .6472979904-1.3591619355$ 
67 C $0.46012423803 .5268745327-1.8913667158$

$68 \mathrm{C}-1.58464120013 .5878483915-0.6078899654$

69 C $-1.58838777394 .9819569221-0.6805871691$

70 C $0.45441435154 .9211388127-1.9653414456$

71 H $1.26349860582 .9703873248-2.3697693889$

$72 \mathrm{H}-2.38523512243 .0792815206-0.0742721512$

$73 \mathrm{H}-2.38679065655 .5466013359-0.2065583757$

74 H $1.24857467235 .4393652083-2.4962635013$

75 H $-0.57283774546 .7327042015-1.4159141218$

Nuclear Repulsion Energy $=4136.9798990219$ hartrees

There are 137 alpha and 137 beta electrons

Requested basis set is $6-31 \mathrm{G}(\mathrm{d})$

There are 228 shells and 657 basis functions

Total QAlloc Memory Limit 1987 MB

Mega-Array Size $188 \mathrm{MB}$

MEM_STATIC part 192 MB

.. (5.1.P)

- Entering fldman on Mon May 10 11:09:50 2021 -

A cutoff of 1.0D-09 yielded 9141 shell pairs

There are 84266 function pairs

Smallest overlap matrix eigenvalue $=1.56 \mathrm{E}-04$

Scale SEOQF with 1.000000e-02/1.000000e-02/1.000000e-02

Standard Electronic Orientation quadrupole field applied

Nucleus-field energy $=-0.0000000005$ hartrees

- Entering gesman on Mon May 10 11:09:51 2021 -

Guess from superposition of atomic densities

Warning: Energy on first SCF cycle will be non-variational

SAD guess density has 274.000001 electrons

- Entering scfman on Mon May 10 11:09:51 2021 -

A restricted hybrid HF-DFT SCF calculation will be performed using Pulay DIIS + Geometric Direct Minimization Exchange: 0.2000 Hartree-Fock +0.0800 Slater +0.7200 B88 Correlation: 0.1900 VWN1RPA + 0.8100 LYP

Using SG-1 standard quadrature grid

SCF converges when RMS gradient is below 1.0E-07

Exchange: 0.2000 Hartree-Fock +0.0800 Slater +0.7200 B88

Correlation: 0.1900 VWN1RPA + 0.8100 LYP

Using SG-1 standard quadrature grid 
\$comment

M0002

\$end

\$molecule

01

$6-0.97589768552-2.7085999704-3.978802363$

$1-0.74544481093-0.59957745212-4.2178990609$

$6-0.77645215549-1.4368459389-3.5325681166$

$6-0.8693511369-3.6779687708-1.7565737886$

$6-0.61007873667-1.2377210252-2.1231139849$

$6-1.0196556377-3.8114904419-3.1042912538$

$6-0.66898318196-2.3598736279-1.2284340521$

$6-0.382290337190 .069682869858-1.5920492939$

$1-0.90074883852-4.5396035801-1.1026880339$

$6-0.228055704680 .25264592017-0.22346696588$

$6-0.30351159548-0.857595253680 .66780214355$

$6-0.51172644349-2.14246779020 .17738277925$

$8-1.2262110127-4.9590482077-3.828439638$

$6-1.3161838359-4.5619233472-5.1997969209$

$8-1.1560357985-3.1420783124-5.2684131519$

$1-2.3011498537-4.8373414092-5.5951680977$

$1-0.51764547016-5.0473789518-5.7734064337$

60.00487157812641 .65500816010 .34175683929

$10.435515182232 .2933823026-0.43593953333$

10.750248228631 .60722031421 .1447743555

$6-0.16878208622-0.612094591112 .1713383372$

$1-0.58922523104-1.4618286352 .7172253904$

$1-0.774405549210 .260728817072 .4478005385$

$6-1.22422968612 .28111682550 .84709652012$

$61.2181934332-0.398107101912 .6071868474$

$6-2.24318661782 .79042501421 .2592006801$

$62.3644550996-0.211926998672 .9527339064$

$6-3.48083585243 .42471702471 .7500192084$

63.75679512950 .0171650848313 .3823572509

$6-3.13151974964 .76321156082 .4407370811$

$1-4.04300741665 .24345532322 .817472835$

$1-2.64364114445 .45012400311 .7411034398$

$1-2.4531571994 .60158006363 .28502273$

$6-4.17571798242 .48586958042 .7639830572$

$1-4.41817178211 .52281605242 .3022561016$

$1-5.10745460762 .94001265923 .1231181937$

$6-4.43055450183 .68855315470 .55809474142$

$1-5.35289230124 .16854206140 .90746070295$

$1-4.69909048262 .75232017340 .056962491816$

$1-3.95843116834 .3457165486-0.17991433665$

$64.5351253988-1.31901240653 .3451948654$

$15.570299853-1.16281629193 .6725644049$

$14.5545698658-1.73293369162 .3312042811$

$14.0728265041-2.05910003024 .0069511436$

63.76116397010 .577303375844 .8237642992

14.79117363980 .750375043575 .1585354151

$13.2867021185-0.124771771755 .5175609099$

13.21698268181 .52610590254 .875883674

64.42860885291 .03285702012 .4290026933 
15.4719140191 .19686632282 .7252269049

13.90903605391 .99659166372 .4541881264

14.4167592270 .66776468381 .3965890065

$1-2.39720477961 .6799981088-2.3248191617$

$6-1.46473656961 .9642673248-2.8039350867$

$6-0.319800520481 .2377728355-2.5321287892$

$6-0.270090499583 .4544325414-4.289214435$

$60.916236680021 .6156061207-3.1559563927$

$6-1.44350506773 .0726760229-3.6831614966$

$60.934448161772 .7431564672-4.0438683047$

$62.13480611390 .91948098493-2.9226366353$

$1-2.36233756063 .6211792966-3.8727011442$

$12.16341658523 .9787876546-5.3248629999$

$1-0.246973459184 .3073947046-4.9635561457$

$63.30541315531 .3132420601-3.5320041888$

$12.1308253110 .066314998495-2.2518118327$

$14.22500826290 .76646128045-3.3404484974$

$63.32096111542 .4254277276-4.4071923571$

$14.25123811262 .7267958929-4.88125064$

$62.16031595993 .1218484238-4.6549076811$

$6-0.55980135554-3.31716442841 .1069100857$

$6-0.63549319485-5.5334947612 .841377658$

$6-1.7744272845-3.95547812861 .4032611696$

$60.61611997628-3.80925253931 .694173647$

$60.57869224535-4.90756987612 .5542244365$

$6-1.8126285902-5.05295779132 .2646764728$

$1-2.6924906011-3.58344614370 .95589119812$

$11.5600873164-3.31617804361 .4800892676$

$11.4999398063-5.27422332412 .9995392993$

$1-2.7632396994-5.53159445282 .4857569353$

$1-0.66501916699-6.3889797433 .5110303251$

$1-3.53127569482 .29544485823 .628938424$

\$end

\$rem

JOBTYPE OPT

TIDY SYM TRUE

METHOD WB97X-D

xc_grid $75000302(75,302)$

BASIS 6-31G(d)

VARTHRESH 2 (default DFT)

INCDFT TRUE (default DFT)

GEOM_OPT_HESSIAN READ (main opt)

EXTERNAL_HESSIAN 1

GUI GUI SPARTAN

TERSE_OUTPUT TRUE

SCF_GÜESS READ

\$end

\$opt

\$end

Standard Nuclear Orientation (Angstroms)

I Atom X Y Z

1 C $-0.9758976855-2.7085999704-3.9788023630$

2 H $-0.7454448109-0.5995774521-4.2178990609$ 
3 C $-0.7764521555-1.4368459389-3.5325681166$ 4 C $-0.8693511369-3.6779687708-1.7565737886$ 5 C $-0.6100787367-1.2377210252-2.1231139849$ 6 C $-1.0196556377-3.8114904419-3.1042912538$ $7 \mathrm{C}-0.6689831820-2.3598736279-1.2284340521$ 8 C $-0.38229033720 .0696828699-1.5920492939$ $9 \mathrm{H}-0.9007488385-4.5396035801-1.1026880339$ 10 C $-0.22805570470 .2526459202-0.2234669659$ 11 C $-0.3035115955-0.85759525370 .6678021436$ 12 C $-0.5117264435-2.14246779020 .1773827793$ 13 O $-1.2262110127-4.9590482077-3.8284396380$ 14 C $-1.3161838359-4.5619233472-5.1997969209$ 15 O $-1.1560357985-3.1420783124-5.2684131519$ $16 \mathrm{H}-2.3011498537-4.8373414092-5.5951680977$ $17 \mathrm{H}-0.5176454702-5.0473789518-5.7734064337$ 18 C 0.00487157811 .65500816010 .3417568393 19 H $0.43551518222 .2933823026-0.4359395333$ 20 H 0.75024822861 .60722031421 .1447743555 21 C $-0.1687820862-0.61209459112 .1713383372$ $22 \mathrm{H}-0.5892252310-1.46182863502 .7172253904$ $23 \mathrm{H}-0.77440554920 .26072881712 .4478005385$ $24 \mathrm{C}-1.22422968612 .28111682550 .8470965201$ 25 C $1.2181934332-0.39810710192 .6071868474$ 26 C -2.24318661782 .79042501421 .2592006801$ 27 C $2.3644550996-0.21192699872 .9527339064$ 28 C -3.48083585243 .42471702471 .7500192084$ 29 C 3.75679512950 .01716508483 .3823572509 30 C -3.13151974964 .76321156082 .4407370811$ $31 \mathrm{H}-4.04300741665 .24345532322 .8174728350$ $32 \mathrm{H}-2.64364114445 .45012400311 .7411034398$ $33 \mathrm{H}-2.45315719904 .60158006363 .2850227300$ $34 \mathrm{C}-4.17571798242 .48586958042 .7639830572$ $35 \mathrm{H}-4.41817178211 .52281605242 .3022561016$ $36 \mathrm{H}-5.10745460762 .94001265923 .1231181937$ 37 C -4.43055450183 .68855315470 .5580947414$ $38 \mathrm{H}-5.35289230124 .16854206140 .9074607030$ $39 \mathrm{H}-4.69909048262 .75232017340 .0569624918$ $40 \mathrm{H}-3.95843116834 .3457165486-0.1799143367$ 41 C $4.5351253988-1.31901240653 .3451948654$ 42 H $5.5702998530-1.16281629193 .6725644049$ 43 H $4.5545698658-1.73293369162 .3312042811$ 44 H $4.0728265041-2.05910003024 .0069511436$ 45 C 3.76116397010 .57730337584 .8237642992 $46 \mathrm{H} 4.79117363980 .75037504365 .1585354151$ 47 H $3.2867021185-0.12477177175 .5175609099$ 48 H 3.21698268181 .52610590254 .8758836740 49 C 4.42860885291 .03285702012 .4290026933 50 H 5.47191401901 .19686632282 .7252269049 51 H 3.90903605391 .99659166372 .4541881264 $52 \mathrm{H} 4.41675922700 .66776468381 .3965890065$ $53 \mathrm{H}-2.39720477961 .6799981088-2.3248191617$ 54 C $-1.46473656961 .9642673248-2.8039350867$ 55 C $-0.31980052051 .2377728355-2.5321287892$ 56 C $-0.27009049963 .4544325414-4.2892144350$ 57 C $0.91623668001 .6156061207-3.1559563927$ 58 C $-1.44350506773 .0726760229-3.6831614966$ 
59 C $0.93444816182 .7431564672-4.0438683047$

60 C $2.13480611390 .9194809849-2.9226366353$

$61 \mathrm{H}-2.36233756063 .6211792966-3.8727011442$

62 H $2.16341658523 .9787876546-5.3248629999$

$63 \mathrm{H}-0.24697345924 .3073947046-4.9635561457$

64 C $3.30541315531 .3132420601-3.5320041888$

65 H $2.13082531100 .0663149985-2.2518118327$

66 H $4.22500826290 .7664612804-3.3404484974$

67 C $3.32096111542 .4254277276-4.4071923571$

68 H $4.25123811262 .7267958929-4.8812506400$

69 C $2.16031595993 .1218484238-4.6549076811$

70 C $-0.5598013555-3.31716442841 .1069100857$

71 C $-0.6354931948-5.53349476102 .8413776580$

72 C $-1.7744272845-3.95547812861 .4032611696$

73 C $0.6161199763-3.80925253931 .6941736470$

74 C $0.5786922453-4.90756987612 .5542244365$

75 C - $1.8126285902-5.05295779132 .2646764728$

$76 \mathrm{H}-2.6924906011-3.58344614370 .9558911981$

77 H $1.5600873164-3.31617804361 .4800892676$

78 H $1.4999398063-5.27422332412 .9995392993$

$79 \mathrm{H}-2.7632396994-5.53159445282 .4857569353$

$80 \mathrm{H}-0.6650191670-6.38897974303 .5110303251$

$81 \mathrm{H}-3.53127569482 .29544485823 .6289384240$

Nuclear Repulsion Energy $=4825.4434217691$ hartrees

There are 150 alpha and 150 beta electrons

Requested basis set is $6-31 \mathrm{G}(\mathrm{d})$

There are 248 shells and 721 basis functions

Total QAlloc Memory Limit 1987 MB

Mega-Array Size $188 \mathrm{MB}$

MEM_STATIC part 192 MB

.. (5.1.P)

- Entering fldman on Mon May 10 10:37:44 2021 -

A cutoff of 1.0D-09 yielded 10201 shell pairs

There are 96401 function pairs

Smallest overlap matrix eigenvalue $=1.54 \mathrm{E}-04$

Scale SEOQF with $1.000000 \mathrm{e}-02 / 1.000000 \mathrm{e}-02 / 1.000000 \mathrm{e}-02$

Standard Electronic Orientation quadrupole field applied

Nucleus-field energy $=-0.0000000049$ hartrees

- Entering gesman on Mon May 10 10:37:45 2021 -

Guess MOs from SCF MO coefficient file

Reading MOs from coefficient file

Reading MOs from coefficient file 
- Entering scfman on Mon May 10 10:37:46 2021 -

Long-range $\mathrm{K}$ will be added via erf

Coulomb attenuation parameter $=0.2$ bohr** $(-1)$

A restricted hybrid HF-DFT SCF calculation will be

performed using Pulay DIIS + Geometric Direct Minimization

Exchange: 0.2220 Hartree-Fock + 1.0000 wB97X-D + LR-HF

Correlation: 1.0000 wB97X-D

Using Euler-Maclaurin-Lebedev $(75,302)$ quadrature formula

Dispersion: Grimme D

SCF converges when RMS gradient is below 1.0E-07

Exchange: 0.2220 Hartree-Fock + 1.0000 wB97X-D + LR-HF

Correlation: $1.0000 \mathrm{wB} 97 \mathrm{X}-\mathrm{D}$

Using Euler-Maclaurin-Lebedev $(75,302)$ quadrature formula

Dispersion: Grimme D

Using Q-Chem read-in guess as SCF_GUESS READ specified. 